\title{
Physical parameters of T dwarfs derived from high-resolution near-infrared spectra ${ }^{\star}$
}

\author{
C. del Burgo ${ }^{1}$, E. L. Martín ${ }^{2,3}$, M. R. Zapatero Osorio ${ }^{2}$, and P. H. Hauschildt ${ }^{4}$
}

\author{
1 School of Cosmic Physics, Dublin Institute for Advanced Studies, Dublin 2, Ireland \\ e-mail: cburgo@cp.dias.ie \\ 2 Instituto de Astrofísica de Canarias, 38200, La Laguna, Tenerife, Spain \\ e-mail: [ege;mosorio]@iac.es \\ 3 University of Central Florida, Physics Department, PO Box 162385, Orlando, FL32816, USA \\ e-mail: ege@physics.ucf.edu \\ 4 Hamburger Sternwarte, Gojenbergsweg 112, 21029 Hamburg, Germany \\ e-mail: yeti@hs.uni-hamburg.de
}

Received 5 August 2008 / Accepted 26 February 2009

\begin{abstract}
Aims. We determine the effective temperature, surface gravity and projected rotational velocity of nine $\mathrm{T}$ dwarfs from the comparison of high-resolution near-infrared spectra and synthetic models, and estimate the mass and age of the objects from state-of-the-art models.

Methods. We use the AMES-COND cloudless solar metallicity models provided by the PHOENIX code to match the spectra of nine T-type field dwarfs observed with the near-infrared high-resolution spectrograph NIRSPEC using ten echelle orders to cover part of the $J$ band from 1.147 to $1.347 \mu \mathrm{m}$ with a resolving power $R \sim 20000$. The projected rotational velocity, effective temperature and surface gravity of the objects are determined based on the minimum root mean square of the differences between the modelled and observed relative fluxes. Estimates of the mass and age of the objects are obtained from effective temperature-surface gravity diagrams, where our results are compared with existing solar metallicity models.

Results. The modelled spectra reproduce quite well the observed features for most of the T dwarfs, with effective temperatures in the range of 922-1009 K, and surface gravities between $10^{4.1}$ and $10^{4.9} \mathrm{~cm} \mathrm{~s}^{-2}$. Our results support the assumption of a dust free atmosphere for $\mathrm{T}$ dwarfs later than $\mathrm{T} 5$, where dust grains form and then gravitationally sediment into the low atmosphere. The modelled spectra do not accurately mimic some individual very strong lines like the K I doublet at 1.2436 and $1.2525 \mu \mathrm{m}$. Our modelled spectra does not match well the observed spectra of the two T dwarfs with earlier spectral types, namely SDSSp J125453.90-012247.4 (T2) and 2MASS J05591914-1404488 (T4.5), which is likely due to the presence of condensate clouds that are not incorporated in the models used here. By comparing our results and their uncertainties to evolutionary models, we estimate masses in the interval $\approx 5-75 M_{\mathrm{J}}$ for $\mathrm{T}$ dwarfs later than T5, which are in good agreement with those found in the literature. We found apparent young ages that are typically between 0.1 and a few Gyr for the same T dwarfs, which is consistent with recent kinematical studies.
\end{abstract}

Key words. stars: fundamental parameters - stars: atmosphers - stars: low-mass, brown dwarfs

\section{Introduction}

The first near-infrared spectrum of a T dwarf (G1 229B) showed a predominance of methane $\left(\mathrm{CH}_{4}\right)$ bands that made it look similar to that of Jupiter (Oppenheimer et al. 1995) despite of a difference in temperature of about $800 \mathrm{~K}$. Atmospheric models were soon developed to fit the spectrum of Gl 229B to estimate its surface gravity, effective temperature, age and mass (Allard et al. 1996; Marley et al. 1996; Tsuji et al. 1996). These models indicated that the atmosphere of this brown dwarf was free from dust grains, which were needed to explain the properties of the warmer L dwarfs (Allard et al. 1997). The dust grains in Gl 229B could be condensed in clouds dominated by organic compounds (Fegley et al. 1996; Griffith et al. 1998). It has also been proposed that a warm dust layer could be present deep inside the photosphere (Tsuji et al. 1999) and that the alkali resonance

* Reduced NIRSPEC spectra of the nine T-type dwarfs are available electronically in fits format at the CDS via anonymous ftp to cdsarc.u-strasbg. $f r(130.79 .128 .5)$ or via http://cdsweb.u-strasbg.fr/cgi-bin/qcat?]/A+A/501/1059 lines have very strong pressure-broadened red wings that provide a significant source of opacity at near-infrared wavelengths (Burrows et al. 2000).

The discovery of free-floating objects similar to Gl 229B (Burgasser et al. 1999; Cuby et al. 1999; Strauss et al. 1999) indicated that these objects are numerous in the solar vicinity. A unified near-infrared classification scheme for T dwarfs has been defined by Burgasser et al. (2006a). Theoretical models provide a good correspondence to the broad-band colors and lowresolution spectra of T dwarfs (Burrows et al. 2006). Currently, the coolest $\mathrm{T}$ dwarfs known have estimated effective temperatures between $600 \mathrm{~K}$ and $700 \mathrm{~K}$ (Warren et al. 2007; Delorme et al. 2008).

Most modelling efforts on $\mathrm{T}$ dwarfs have concentrated on fitting broad-band colors and low-resolution spectra, and deriving atmospheric parameters by comparing the data with synthetic spectra (Cushing et al. 2008; Leggett et al. 2007; Saumon et al. 2007, 2006; Burgasser et al. 2006b; Tsuji et al. 2005, 2004; Burgasser et al. 2004). Martín \& Zapatero Osorio (2003) estimated the surface gravity and effective temperature of one 
T dwarf from a mid-resolution near-infrared spectrum. Highresolution $(R \sim 20000)$ observations in the $J$-band obtained with NIRSPEC on the Keck II telescope are now available (Zapatero Osorio et al. 2006; Mc Lean et al. 2007).

Modelling the atmospheres and spectra of $\mathrm{T}$ dwarfs presents a set of challenges. In the AMES-COND models used in this work (Allard et al. 2001), the profile of individual strong IR lines are approximated by Voigt profiles with estimated damping constants as no better data are currently available for these lines. The line databases for molecules such as $\mathrm{FeH}, \mathrm{CaH}, \mathrm{CH}_{4}$ are not complete and in many cases lack accuracy. However, in general the molecular line data are quite good as data sources such as HITRAN are of high quality. The lower the effective temperatures are, the better the completeness of the molecular line data as the (comparatively) less accurate higher energy levels are less populated and thus have smaller effects at lower temperatures.

In the AMES-COND models a dust-free configuration is used, where the dust particles form but do not contribute to the opacities (Allard et al. 2001). This assumption should be reasonable for cool T dwarfs, but will become progressively worse for effective temperatures above $\approx 1200 \mathrm{~K}$. Overall, the systematic errors are still considerably larger than, e.g., for solar type stars, but the situation can only improve if more comparison between models and data are made to identify the areas where improvements are most urgently needed.

In this paper, we present the first comparison with AMESCOND models of high-resolution near-infrared spectra of a sample of nine T dwarfs observed by Zapatero Osorio (2006) that are used to derive their physical parameters. A summary of the observations used here is given in Sect. 2. Section 3 is devoted to the analysis of the data. The results are presented in Sect. 4. Section 5 contains the discussion of our results, and finally, Sect. 6 contains our conclusions.

\section{Observations, models and data preparation}

\subsection{Observations}

High-resolution near-infrared spectra in the $J$-band of nine $\mathrm{T}$ dwarfs were obtained using the Keck II telescope and the NIRSPEC spectrograph. The instrumental setup was chosen to provide a wavelength coverage from 1.148 up to $1.346 \mu \mathrm{m}$ split into ten different orders, a nominal dispersion ranging from 0.164 (blue wavelengths) to $0.191 \AA \mathrm{pix}^{-1}$ (red wavelengths), and a final resolution element of $0.55-0.70 \AA$ at $1.2485 \mu \mathrm{m}$ (roughly the central wavelength of the spectra), corresponding to a resolving power $R=17800-22700$. A detailed description of the observations and data reduction is provided in Zapatero Osorio et al. (2006, hereinafter ZO06), who presented the T dwarfs that we used in this study.

In our spectroscopic analysis we have used as many echelle orders as possible: from order 57 up to order 66; this depends on the quality (in terms of signal-to-noise ratio) of the observed spectra. Table 2 in McLean et al. (2007) summarizes the wavelength and dispersion properties of each of the $J$-band NIRSPEC echelle orders 58 to 65 for the same instrumental configuration that we have used here. Order 57 covers the wavelength interval 1.327-1.347 $\mu \mathrm{m}$ with a nominal dispersion of $0.191 \AA \mathrm{pix}^{-1}$. Order 66 covers the range $1.147-1.164 \mu \mathrm{m}$ with a nominal dispersion of $0.167 \AA$ pix $^{-1}$.

Figure 1 shows, as an example, the order 61 with the presence of the KI doublet at 1.2436 and $1.2525 \mu \mathrm{m}$, for all the $\mathrm{T}$ dwarfs.

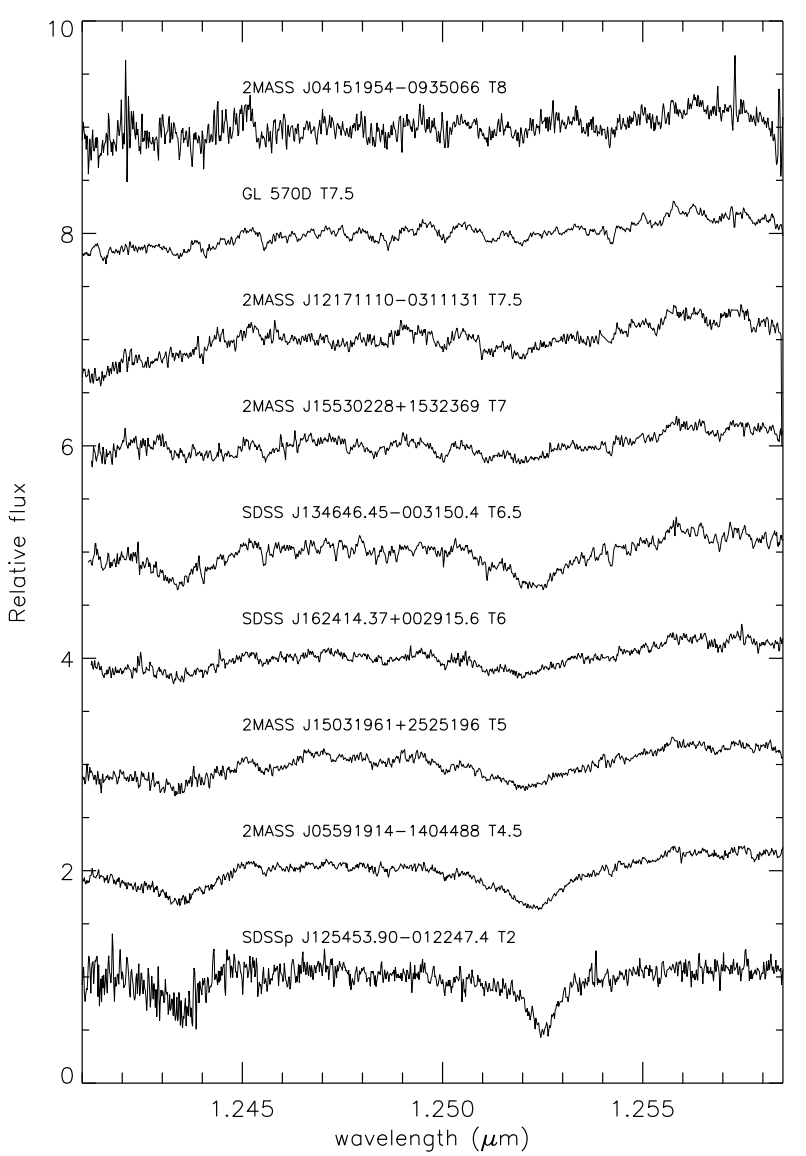

Fig. 1. Variation of the strong K I doublet at 1.2436 and $1.2525 \mu \mathrm{m}$ along the spectral type sequence of T dwarfs. All spectra are normalized to unity over the entire wavelength range and offset by a constant. Also, each spectrum is shifted in velocity to vacuum wavelengths.

\subsection{Description of atmosphere models}

The PHOENIX code (Hauschildt \& Baron 1999) is a general purpose stellar atmosphere modelling package that includes very complex atomic models and line blanketing by hundreds of millions of atomic and molecular lines. The code can be used to compute model atmospheres and synthetic spectra of cool objects. The radiative transfer in PHOENIX is solved in spherical geometry using an operator splitting method (Hauschildt 1992, 1993). At the low temperatures of the brown dwarfs, a rich chemistry in the atmospheres of these objects is present, with hundreds of gas-phase species, liquids and crystals, and the formation of tens of different types of dust grains (e.g., silicates, amorphous carbon, iron). There are four different scenarios for the dust formation considered in PHOENIX, among which is the AMES-COND cloudless models (Allard et al. 2001).

Here we used a grid of the AMES-COND v2.2 models and synthetic spectra of solar metallicity, with effective temperatures $T_{\text {eff }}$ ranging from 700 to $3000 \mathrm{~K}$ (steps of $100 \mathrm{~K}$ ) and surface gravities $\log g$ ranging from 3.0 to 5.5 (steps of 0.5 ) with $g$ in $\mathrm{cm} \mathrm{s}^{-2}$, to mimic the observed spectra of $\mathrm{T}$ dwarfs.

The AMES-COND v2.2 models use the $\mathrm{H}_{2} \mathrm{O}$ line list of Barber et al. (2006), the FeH list of Dulick et al. (2003), and the damping constants used for the K I lines are published in Allard et al. (2003).

Figure 2 illustrates the identification of some near-infrared features over a synthetic spectrum with $v_{\text {rot }} \sin i=0, T_{\text {eff }}=$ $1000 \mathrm{~K}$ and $\log g=4.5$. Most features are due to water vapor, 

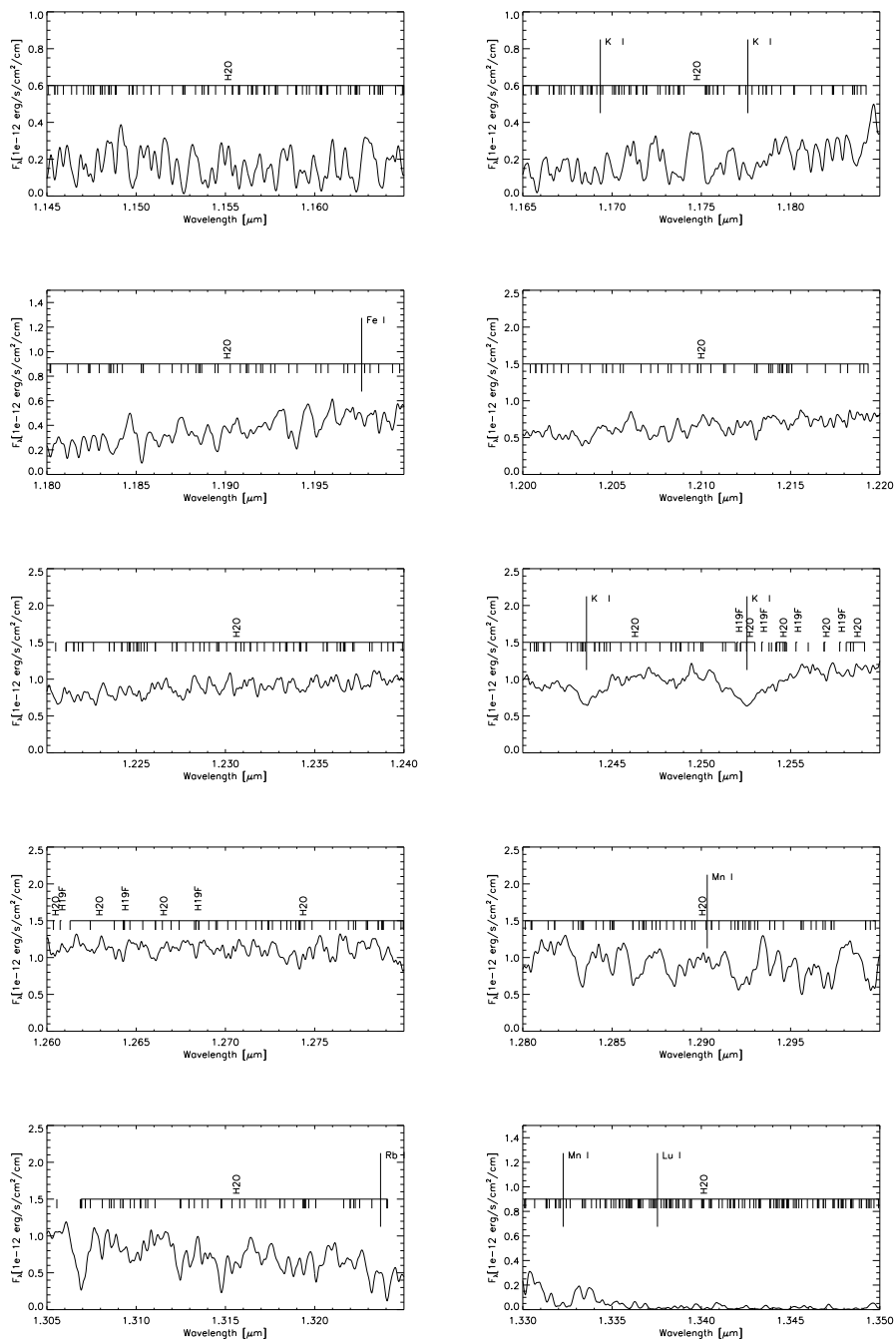

Fig. 2. Synthetic flux versus wavelength. Identifications of some features of a synthetic spectrum with $T_{\text {eff }}=1000 \mathrm{~K}$ and $\log g=4.5$. Most of the lines are due to water vapor.

and the only visible atomic lines are due to the $\mathrm{K}$ I doublet at $1.25 \mu \mathrm{m}$.

Figure 3 shows the variations of the synthetic spectra for a surface gravity of $\log g=4.5$ and different effective temperatures from 700 to $1100 \mathrm{~K}$. We note the strong change of the $\mathrm{K}$ I doublet at 1.2436 and $1.2525 \mu \mathrm{m}$ with temperature. Figure 4 shows the variations of the synthetic spectra for various $\log g$ (from 3.5 to 5.5 ) and the same $T_{\text {eff }}=1000 \mathrm{~K}$.

\subsection{Data preparation}

All of our observed spectra were moved to vacuum wavelengths (i.e., laboratory frame of reference) for a proper comparison with the theoretical models. This was done from a cross-correlation analysis. In a first iteration, we used the radial velocities published in Zapatero Osorio et al. (2007). Then, we determined the atmospheric parameters for each $\mathrm{T}$ dwarf as explained later and used the corresponding synthetic spectra as templates to recompute the radial velocities. New atmospheric parameters were thus obtained consistently with the recalculated radial velocities. We note that our radial velocities are in agreement, within the errors, with those of Zapatero Osorio et al. (2007).

The grid of PHOENIX synthetic spectra were modified in order to be compared with the NIRSPEC observations. First,
Table 1. SDSSp J125453.90-012247.4. Synthetic and observed spectra comparison for all orders: rms, $N, T_{\text {eff }}, \log g$ and $v_{\text {rot }} \sin i$.

\begin{tabular}{lccccc}
\hline \hline Order & $N$ & rms & $T_{\text {eff }}$ & $\log g$ & $v_{\text {rot }} \sin i$ \\
\hline 66 & 996 & 0.2797 & 1600 & 5.5 & 38 \\
65 & 995 & 0.1740 & 1300 & 5.5 & 46 \\
64 & 1001 & 0.1336 & 2400 & 3.5 & 36 \\
63 & 1002 & 0.0931 & 2500 & 3.5 & 26 \\
62 & 1002 & 0.0839 & 2700 & 3.5 & 27 \\
61 & 1002 & 0.0960 & 2700 & 5.5 & 46 \\
60 & 890 & 0.0914 & 700 & 5.5 & 28 \\
59 & 1002 & 0.0811 & 2000 & 3.0 & 36 \\
58 & 1002 & 0.0932 & 1500 & 5.5 & 36 \\
57 & 994 & 0.0862 & 2000 & 4.0 & 31 \\
\hline
\end{tabular}

Table 2. 2MASS J05591914-1404488. Synthetic and observed spectra comparison for all orders: rms, $N, T_{\mathrm{eff}}, \log g$ and $v_{\mathrm{rot}} \sin i$.

\begin{tabular}{lccccc}
\hline \hline Order & $N$ & rms & $T_{\text {eff }}$ & $\log g$ & $v_{\text {rot }} \sin i$ \\
\hline 66 & 987 & 0.1079 & 1500 & 5.0 & 21 \\
65 & 989 & 0.0796 & 1200 & 5.5 & 25 \\
64 & 991 & 0.0575 & 1000 & 3.5 & 33 \\
63 & 991 & 0.0512 & 800 & 5.5 & 24 \\
62 & 991 & 0.0416 & 800 & 5.5 & 27 \\
61 & 991 & 0.0612 & 1000 & 5.0 & 33 \\
60 & 882 & 0.0434 & 800 & 5.5 & 25 \\
59 & 991 & 0.0724 & 900 & 3.5 & 33 \\
58 & 991 & 0.0682 & 1300 & 5.5 & 33 \\
57 & 957 & 0.0491 & 1500 & 4.0 & 33 \\
\hline
\end{tabular}

the synthetic spectra were transformed to take into account the projected rotational velocity $\left(v_{\text {rot }} \sin i\right)$ of the objects using the formalism of Gray (1992), with a limb darkening parameter $\epsilon=0.6$. A grid of models with projected rotational velocities between 10 and $50 \mathrm{~km} \mathrm{~s}^{-1}$, with steps of $1 \mathrm{~km} \mathrm{~s}^{-1}$, were generated. These spectra were also convolved with a Gaussian that mimics the instrumental profile along the dispersion axis for each order. The resulting modelled spectra were rebinned to the same resolution of the observations. Modelled spectra are normalized over the wavelength range corresponding to order 59. All these steps were performed using our own programmes.

\section{Analysis}

In order to constrain the number of possible solutions provided by our large set of models, the root-mean-square $\operatorname{rms}\left(v_{\text {rot }} \sin i\right.$, $T_{\text {eff }}, \log g$ ) is obtained for each model:

$\operatorname{rms}\left(v_{\mathrm{rot}} \sin i, T_{\mathrm{eff}}, \log g\right)=\sqrt{\frac{1}{N} \sum_{i=1}^{n}\left(F_{v}(i)-G_{\nu}(i)\right)^{2}}$,

where $i$ stands for $i$-pixel in the spectral axis, $F_{v}(i)$ and $G_{v}(i)$ correspond to the observed and modelled fluxes, respectively. We compute values of rms for the whole set of models and for all echelle orders independently, since different orders show different signal-to-noise ratios.

Tables 1-9 show the values of $T_{\text {eff }}, \log g$, and $v_{\text {rot }}$ corresponding to the model that minimizes the rms for each echelle order. The total number of pixels $N$ used in the comparison is also listed. For order 60, the wavelength range between 1.268 and $1.270 \mu \mathrm{m}$ is excluded from the comparison because of the presence of strong telluric lines that practically blocked any signal from the targets. To avoid border effects, a few pixels at both sides of each echelle order have been rejected. 


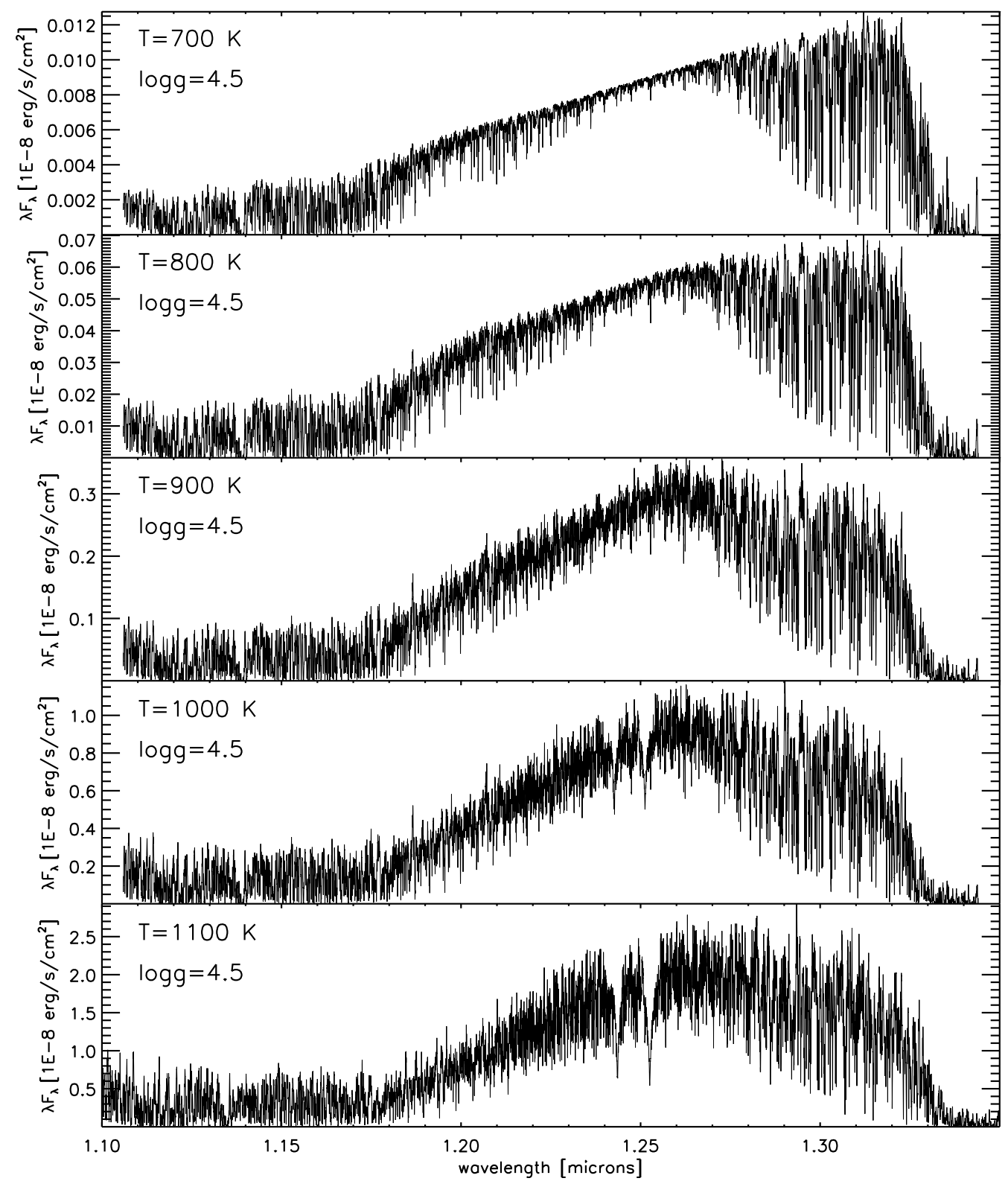

Fig. 3. Synthetic flux versus wavelength. Models with $\log g=4.5$ (cgs units) and $T_{\text {eff }}=700,800,900,1000$ and $1100 \mathrm{~K}$. The synthetic spectra were computed with $0.05 \AA$ pix $^{-1}$, but are shown after being smoothed with a boxcar average of 10 pixel width.

Table 3. 2MASS J15031961+2525196. Synthetic and observed spectra comparison for all orders: rms, $N, T_{\text {eff }}, \log g$ and $v_{\text {rot }} \sin i$.

\begin{tabular}{lccccc}
\hline \hline Order & $N$ & rms & $T_{\text {eff }}$ & $\log g$ & $v_{\text {rot }} \sin i$ \\
\hline 66 & 969 & 0.1112 & 1300 & 5.0 & 34 \\
65 & 978 & 0.0627 & 1100 & 4.0 & 42 \\
64 & 987 & 0.0542 & 900 & 4.0 & 30 \\
63 & 987 & 0.0445 & 1000 & 3.5 & 42 \\
62 & 987 & 0.0413 & 800 & 5.5 & 37 \\
61 & 987 & 0.0576 & 1000 & 5.5 & 42 \\
60 & 873 & 0.0461 & 800 & 5.5 & 31 \\
59 & 987 & 0.0619 & 900 & 3.5 & 37 \\
58 & 987 & 0.0633 & 1300 & 5.0 & 33 \\
57 & 911 & 0.0448 & 1400 & 4.5 & 33 \\
\hline
\end{tabular}

\subsection{Average physical parameters}

We compute the average values of $T_{\text {eff }}, \log g$, and $v_{\text {rot }}$ for each object in our sample by combining the various values obtained
Table 4. SDSS J162414.37+002915.6. Synthetic and observed spectra comparison for all orders: rms, $N, T_{\text {eff }}, \log g$ and $v_{\text {rot }} \sin i$.

\begin{tabular}{lccccc}
\hline \hline Order & $N$ & rms & $T_{\text {eff }}$ & $\log g$ & $v_{\text {rot }} \sin i$ \\
\hline 64 & 977 & 0.0396 & 1000 & 4.0 & 43 \\
63 & 977 & 0.0437 & 1000 & 4.5 & 47 \\
62 & 977 & 0.0386 & 800 & 5.0 & 41 \\
61 & 976 & 0.0550 & 900 & 5.5 & 47 \\
60 & 858 & 0.0533 & 900 & 3.5 & 37 \\
59 & 977 & 0.0582 & 1100 & 5.0 & 40 \\
58 & 977 & 0.0671 & 1300 & 5.0 & 35 \\
\hline
\end{tabular}

for the different echelle orders according to the following equations:

$\left\langle T_{\mathrm{eff}}\right\rangle=\frac{\sum_{j}\left(T_{\mathrm{eff}, j} \frac{W_{j}}{\sigma_{j}}\right)}{\sum_{j} \frac{W_{j}}{\sigma_{j}}}$, 


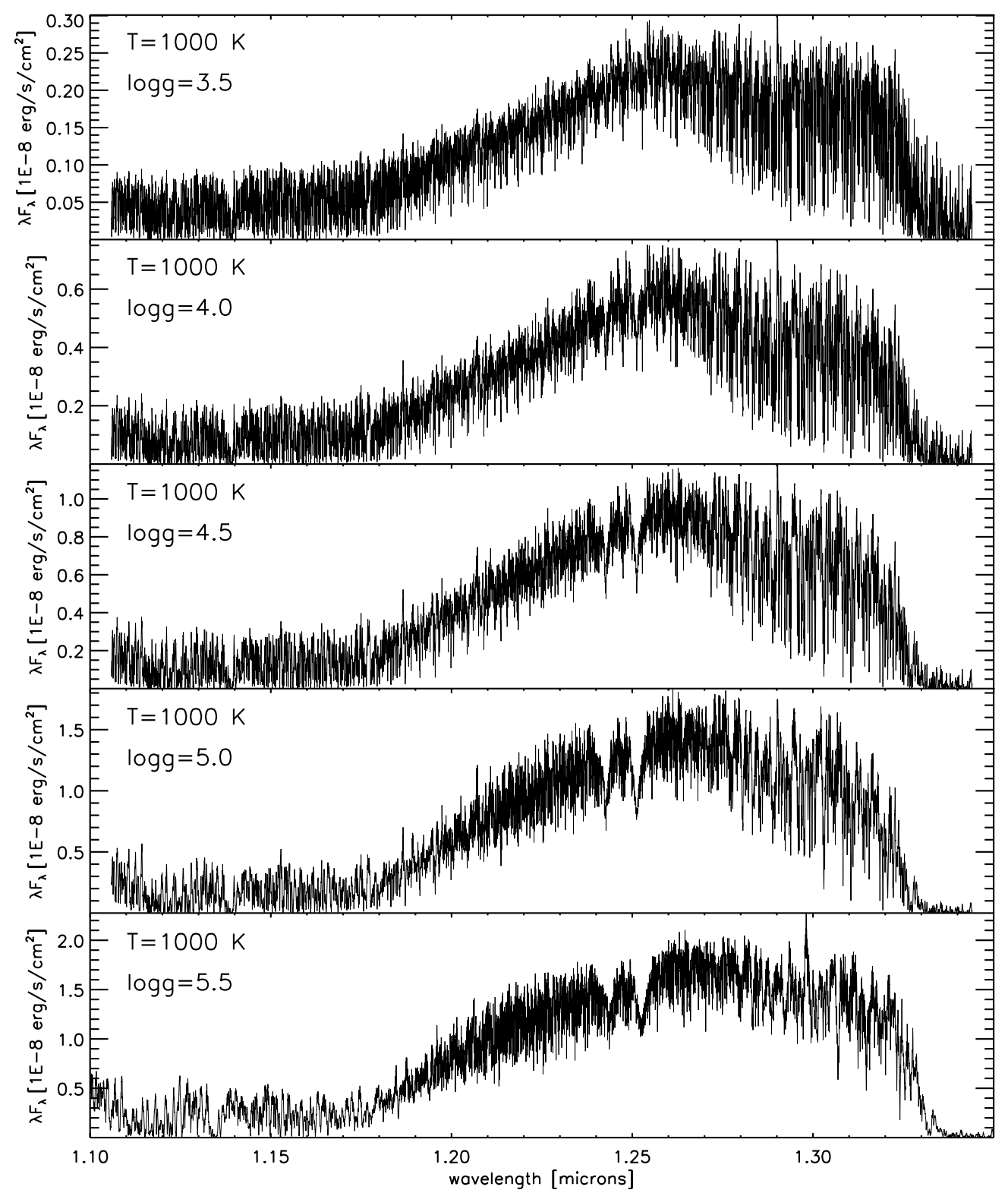

Fig. 4. Synthetic flux versus wavelength. Models with $T_{\text {eff }}=1000 \mathrm{~K}$ and $\log g=3.5,4.0,4.5,5.0$ and 5.5 (cgs units). The synthetic spectra were computed with $0.05 \AA$ pix $^{-1}$, but are shown after being smoothed with a boxcar average of 10 pixel width.

Table 5. SDSS J134646.45-003150.4. Synthetic and observed spectra comparison for all orders: rms, $N, T_{\text {eff }}, \log g$ and $v_{\text {rot }} \sin i$.

\begin{tabular}{lccccc}
\hline \hline Order & $N$ & rms & $T_{\text {eff }}$ & $\log g$ & $v_{\text {rot }} \sin i$ \\
\hline 64 & 972 & 0.0544 & 1000 & 4.0 & 19 \\
63 & 974 & 0.0554 & 1000 & 4.0 & 20 \\
62 & 974 & 0.0500 & 900 & 4.5 & 17 \\
61 & 974 & 0.0644 & 1000 & 5.0 & 25 \\
60 & 862 & 0.0801 & 900 & 4.0 & 12 \\
59 & 974 & 0.1185 & 900 & 4.0 & 13 \\
58 & 973 & 0.1139 & 1200 & 4.5 & 13 \\
\hline
\end{tabular}

$$
\begin{gathered}
\langle\log g\rangle=\frac{\sum_{j}\left(\log g_{j} \frac{W_{j}}{\sigma_{j}}\right)}{\sum_{j} \frac{W_{j}}{\sigma_{j}}}, \\
\left\langle v_{\mathrm{rot}} \sin i\right\rangle=\frac{\sum_{j}\left(v_{\mathrm{rot}} \sin i_{j} \frac{W_{j}}{\sigma_{j}}\right)}{\sum_{j} \frac{W_{j}}{\sigma_{j}}},
\end{gathered}
$$

Table 6. 2 MASS J15530228+1532369. Synthetic and observed spectra comparison for all orders: rms, $N, T_{\text {eff }}, \log g$ and $v_{\text {rot }} \sin i$.

\begin{tabular}{lccccc}
\hline \hline Order & $N$ & rms & $T_{\text {eff }}$ & $\log g$ & $v_{\text {rot }} \sin i$ \\
\hline 63 & 976 & 0.0507 & 1000 & 4.5 & 37 \\
62 & 976 & 0.0582 & 800 & 5.0 & 21 \\
61 & 976 & 0.0659 & 900 & 5.5 & 39 \\
60 & 857 & 0.0563 & 900 & 4.0 & 27 \\
59 & 976 & 0.0733 & 900 & 4.0 & 30 \\
58 & 979 & 0.0863 & 1100 & 5.0 & 23 \\
\hline
\end{tabular}

where $\sigma_{j}$ refers to the minimum rms for the echelle order $j$ (as listed in Tables 1-9), and $W_{j}$ corresponds to the weight that we have assigned to the echelle order $j$ based on the signal-to-noise ratio of the observations. Table 10 shows the various $W_{j}$, which are relative to order 61 . Regarding the echelle order 60, we have given lower weights to J1217-0311 and J0415-0935 because the correction for Earth telluric lines were not optimal. Finally, the average values of $T_{\text {eff }}, \log g$, and $v_{\text {rot }}$ are provided in Table 11, 
Table 7. 2MASS J12171110-0311131. Synthetic and observed spectra comparison for all orders: rms, $N, T_{\text {eff }}, \log g$ and $v_{\text {rot }} \sin i$.

\begin{tabular}{lccccc}
\hline \hline Order & $N$ & rms & $T_{\text {eff }}$ & $\log g$ & $v_{\text {rot }} \sin i$ \\
\hline 63 & 843 & 0.0467 & 800 & 5.5 & 21 \\
62 & 972 & 0.0581 & 900 & 4.0 & 32 \\
61 & 972 & 0.0947 & 900 & 5.5 & 41 \\
60 & 882 & 0.1777 & 900 & 4.0 & 21 \\
59 & 972 & 0.0894 & 1000 & 4.5 & 27 \\
58 & 972 & 0.0918 & 1100 & 4.5 & 29 \\
\hline
\end{tabular}

Table 8. GL 570D. Synthetic and observed spectra comparison for all orders: rms, $N, T_{\text {eff }}, \log g$ and $v_{\text {rot }} \sin i$.

\begin{tabular}{lccccc}
\hline \hline Order & $N$ & rms & $T_{\text {eff }}$ & $\log g$ & $v_{\text {rot }} \sin i$ \\
\hline 64 & 974 & 0.0327 & 900 & 4.5 & 27 \\
63 & 974 & 0.0411 & 1000 & 4.5 & 34 \\
62 & 974 & 0.0428 & 1000 & 4.0 & 41 \\
61 & 974 & 0.0649 & 900 & 5.5 & 41 \\
60 & 861 & 0.0675 & 900 & 4.0 & 26 \\
59 & 974 & 0.0919 & 1000 & 4.5 & 26 \\
58 & 974 & 0.0986 & 1000 & 5.0 & 21 \\
\hline
\end{tabular}

Table 9. 2MASS J04151954-0935066. Synthetic and observed spectra comparison for all orders: rms, $N, T_{\text {eff }}, \log g$ and $v_{\text {rot }} \sin i$.

\begin{tabular}{lccccc}
\hline \hline Order & $N$ & rms & $T_{\text {eff }}$ & $\log g$ & $v_{\text {rot }} \sin i$ \\
\hline 64 & 926 & 0.2029 & 800 & 3.5 & 43 \\
63 & 981 & 0.1791 & 1000 & 3.5 & 33 \\
62 & 982 & 0.1119 & 1000 & 4.0 & 44 \\
61 & 987 & 0.1206 & 900 & 5.0 & 43 \\
60 & 899 & 0.1539 & 1000 & 3.5 & 38 \\
59 & 987 & 0.1081 & 900 & 4.5 & 29 \\
58 & 984 & 0.1330 & 1000 & 5.0 & 25 \\
\hline
\end{tabular}

Table 10. Weights of echelle orders.

\begin{tabular}{lcc}
\hline \hline Order & Weight & Remark \\
\hline 66 & 0.3 & for all objects \\
65 & 0.4 & for all objects \\
64 & 0.6 & for all objects \\
63 & 1.0 & for all objects \\
62 & 1.0 & for all objects \\
61 & 1.0 & for all objects \\
60 & 0.2 & for 2MASS J12171110-0311131 \\
& 0.6 & for 2MASS J04151954-0935066 \\
& 1.0 & for the rest \\
59 & 1.3 & for all objects \\
58 & 1.1 & for all objects \\
57 & 0.8 & for all objects \\
\hline
\end{tabular}

where we also include the spectral types of the targets following the unified scheme of Burgasser et al. (2006a). Errors in $\left\langle T_{\text {eff }}\right\rangle$, $\langle\log g\rangle$ and $\left\langle v_{\text {rot }} \sin i\right\rangle$ were obtained from the standard deviation of the corresponding values derived for the various echelle orders. We note that if no weights are taken into account, the solutions for $\left\langle T_{\mathrm{eff}}\right\rangle,\langle\log g\rangle$ and $\left\langle v_{\text {rot }} \sin i\right\rangle$ do not change significantly, with differences with respect of those obtained using the weights $W_{j}$ of $\pm 40 \mathrm{~K}$ (for T dwarfs later than $\mathrm{T} 5$ the difference is only $\pm 7 \mathrm{~K}), \pm 0.1 \mathrm{dex}$, and $\pm 1 \mathrm{~km} \mathrm{~s}^{-1}$, which are much smaller than the quoted uncertainties.

Figure 5 shows, as an example, some contours of equal rms (Root mean square) around the solution $\left(\left\langle T_{\text {eff }}\right\rangle, \log g\right)$ for GL570D, with $v_{\text {rot }}=32 \mathrm{~km} \mathrm{~s}^{-1}$. The isocontour of the rms of 1.25 times the minimum rms enclose values of $\log g$ of

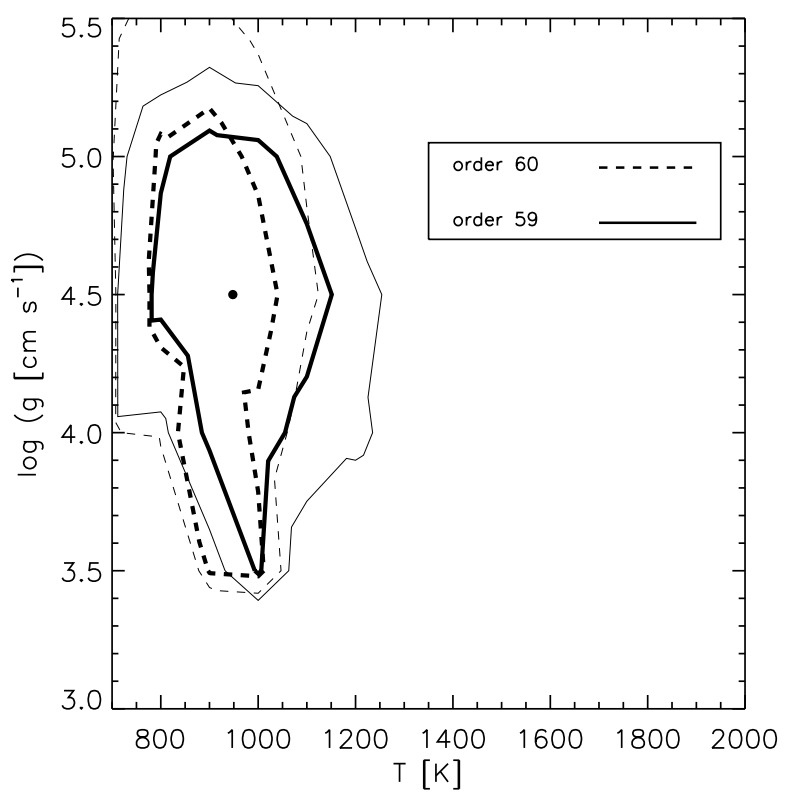

Fig. 5. Contours with $\mathrm{rms}=1.25$ (thick lines) and 1.5 (thin lines) times the value of the minimum rms for orders 59 (solid lines) and 60 (dashed lines). Location of $\left(\left\langle T_{\mathrm{eff}}\right\rangle, \log g\right)$ for the minimum rms is also indicated (dot).

$\approx \pm 0.7 \mathrm{dex}$ and $T_{\text {eff }}$ of $\approx \pm 150 \mathrm{~K}$ around the solution. In general, the isocontours of the rms enclose a unique solution with a broad range of values of gravity and temperature around the solution.

\subsection{Best modelled spectra}

We have computed the modelled spectra determined from the average effective physical parameters determined in Sect. 3.1 (hereinafter, best modelled spectra). We have used a bilinear interpolation to compute the synthetic models corresponding to $\left\langle T_{\text {eff }}\right\rangle$ and $\langle\log g\rangle$ from our AMES-COND model grid. This bilinear interpolation has been done using the logarithm of the synthetic fluxes. We first proved that a linear interpolation in both axes was appropriate. We fixed $T_{\text {eff }}$ at $1000 \mathrm{~K}$ and used the corresponding synthetic models at $\log g=4.0$ and $\log g=5.0$ to compute interpolated spectra at $\log g=4.5$. We also fixed $\log g=4.5$ and used the corresponding synthetic models at $T_{\mathrm{eff}}=900 \mathrm{~K}$ and $1100 \mathrm{~K}$ to compute the interpolated spectra at $T_{\text {eff }}=1000 \mathrm{~K}$. We then applied the same steps described in Sect. 2.3 to compare the synthetic spectra to our observations. The differences between the interpolated and synthetic models are typically of a few per cent. The largest differences of $10 \%$ observed between the spectra were obtained for the echelle order 57 with a null rotational broadening. For the rest of the echelle orders the differences were generally below $1 \%$.

Figures 6-14 show the observations and modelled spectra for our list of T dwarfs.

\section{Results}

\subsection{Near-infrared absorption features of $T d$ warfs}

As observed by McLean et al. (2007), the $J$-band spectral morphology at $R \sim 20000$ of $\mathrm{T}$ dwarfs shows a dense population of weak absorption features and a few relatively strong lines. The fine-scale spectral structure is mainly associated with $\mathrm{H}_{2} \mathrm{O}$, and the strong atomic features are doublets of $\mathrm{KI}$ that appear in orders 61 and 65 . The strong $\mathrm{KI}_{\mathrm{I}}$ doublet in order 61, at 

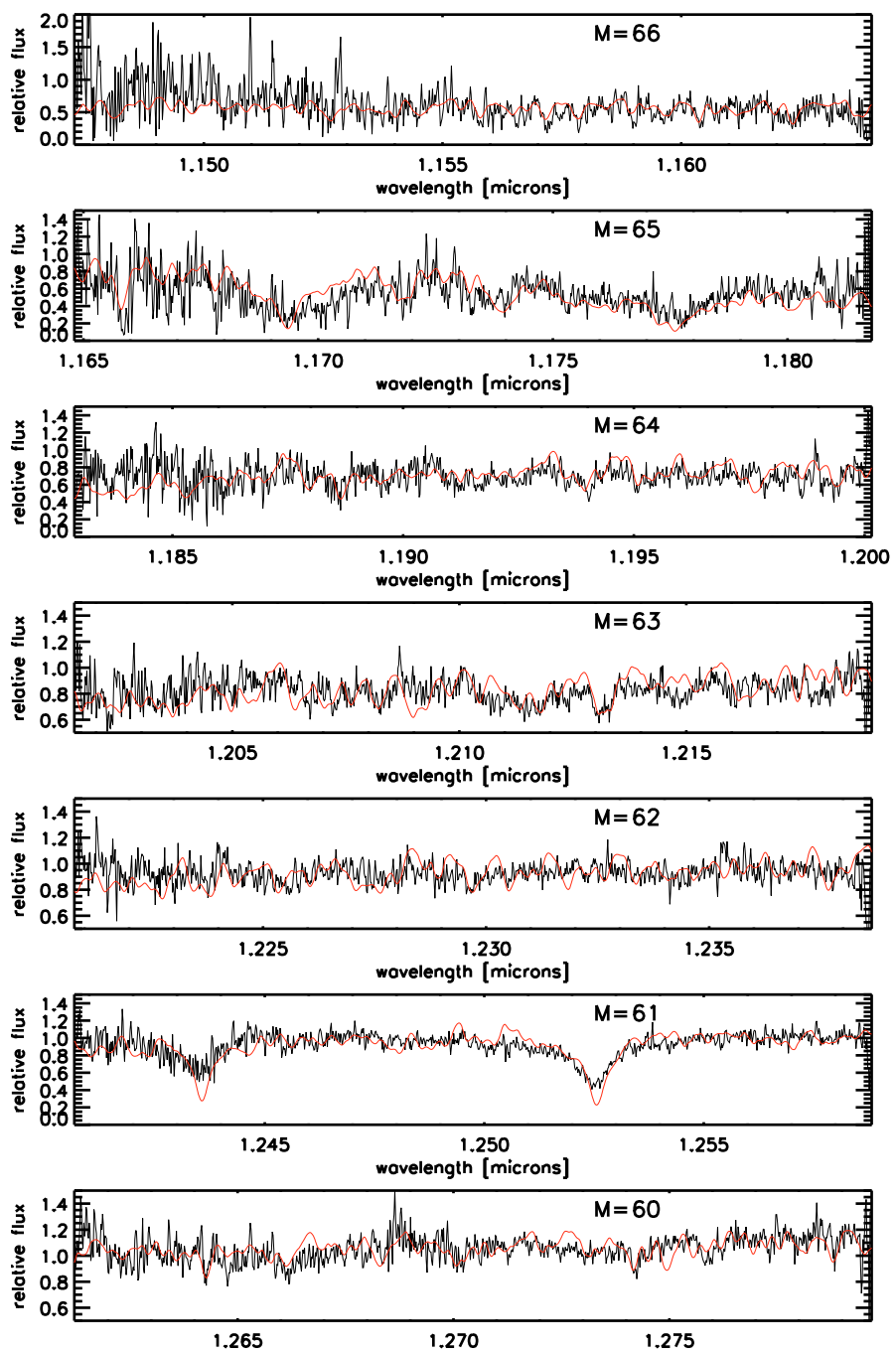

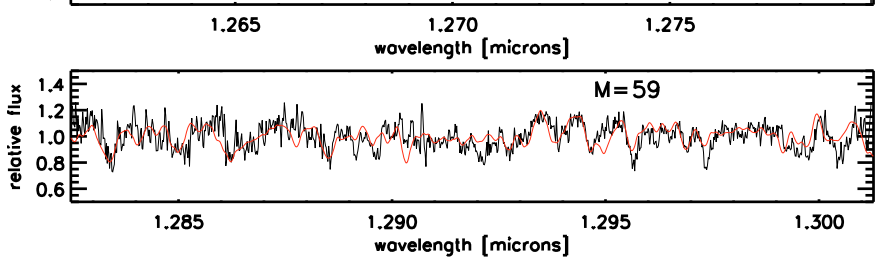
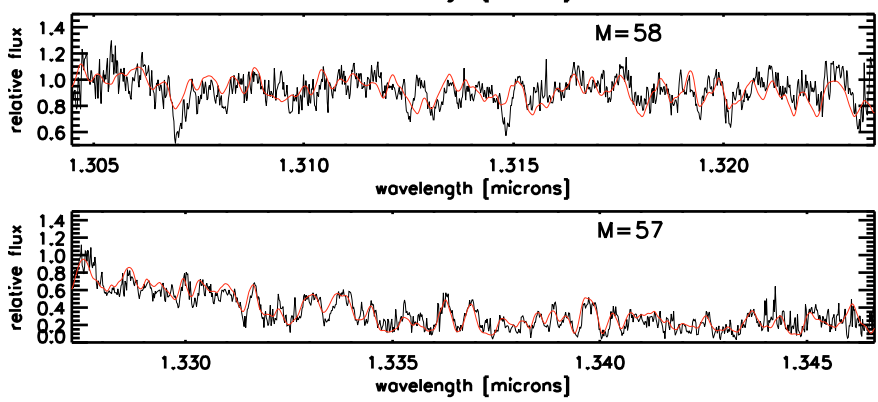

Fig. 6. SDSSp J125453.90-012247.4. Black and red lines correspond to the observed and modelled spectra, respectively. The model is for an effective temperature of $2000 \mathrm{~K}$ and a surface gravity of $10^{4.3} \mathrm{~cm} \mathrm{~s}^{-2}$.

wavelengths 1.2436 and $1.2525 \mu \mathrm{m}$, deepen towards earlier Ts (see Fig. 1). We note that the lines of this doublet in SDSS J134646.45-003150.4 are stronger than what would be expected according to its spectral type (T6.5).

$\mathrm{FeH}$ lines in orders 62 and 63 are weaker towards later Ts. Some absorption around $1.222 \mu \mathrm{m}$ may be present even at T4.5. Those at 1.24637 and $1.24825 \mu \mathrm{m} \mathrm{FeH}$ in order 61 are not visible
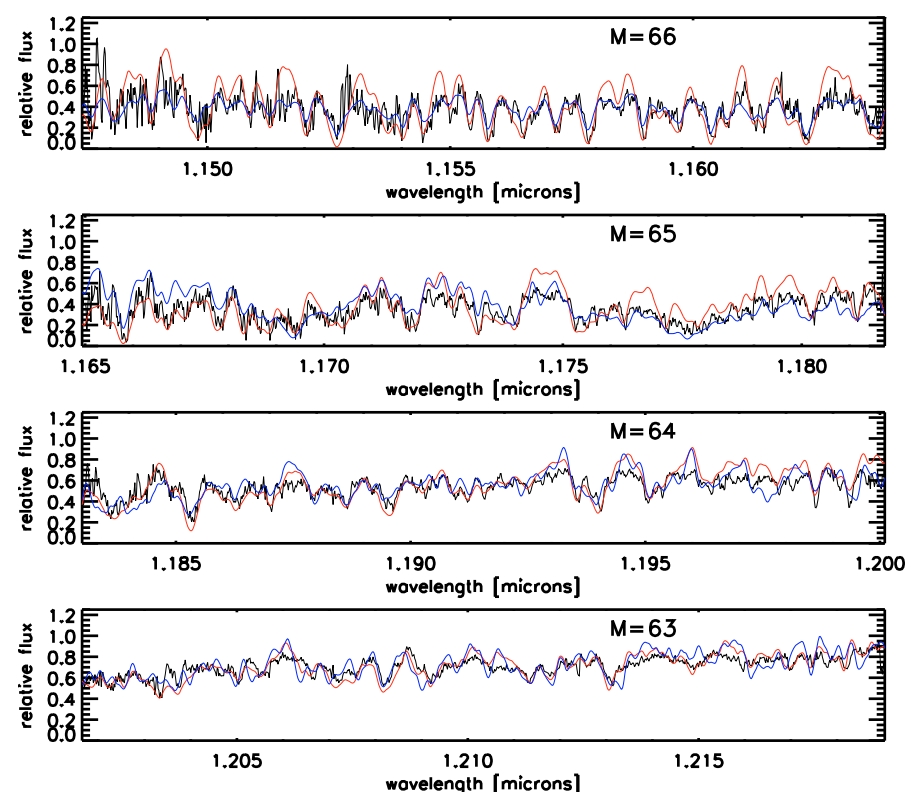

$\frac{\underbrace{}_{0.6}}{\frac{1.225}{\text { movelength [microns] }}}$

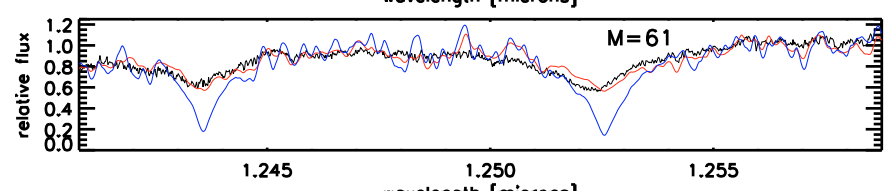

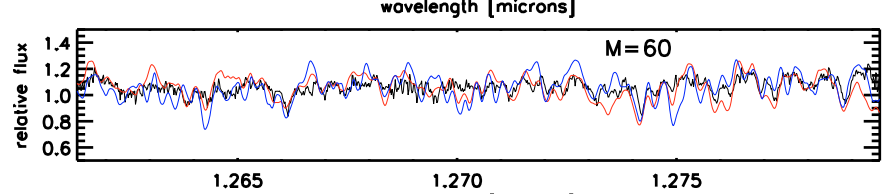
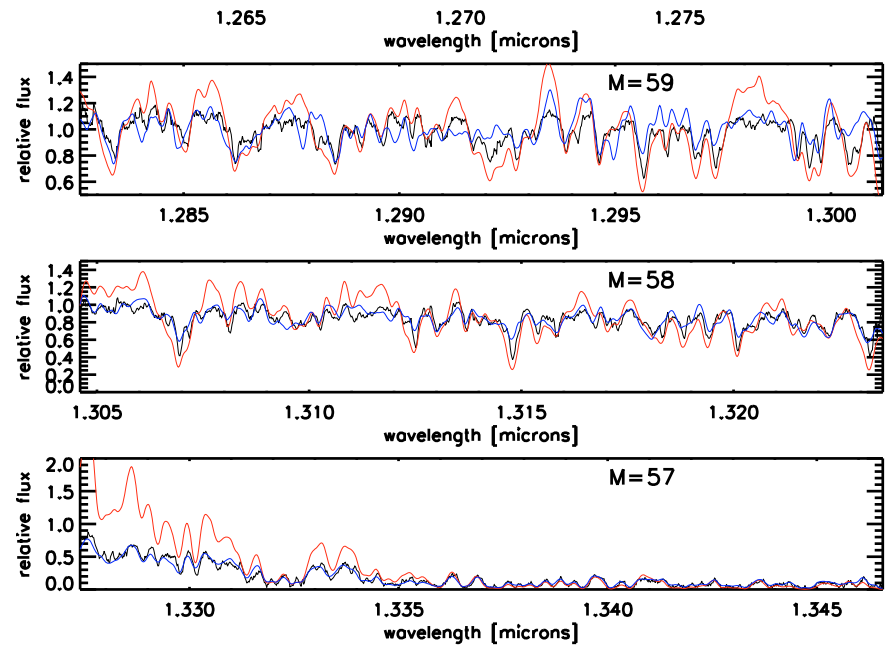

Fig. 7. 2MASS J05591914-1404488. Black line corresponds to the observed spectrum, and red and blue lines correspond to modelled spectra with the same $g=10^{4.9} \mathrm{~cm} \mathrm{~s}^{-2}$ and $T_{\text {eff }}=1002$, and $1700 \mathrm{~K}$.

in T dwarfs. Order 64 is dominated by sharp and deep $\mathrm{H}_{2} \mathrm{O}$ absorption features. This is also the case of order 66 , and more dramatically for order 57 , with the strongest $\mathrm{H}_{2} \mathrm{O}$ absorption in the observed range. Order 65 shows the other K I doublet and changes with spectral type. It also contains many strong intrinsic transitions of hot $\mathrm{H}_{2} \mathrm{O}$, like the feature at $1.175 \mu \mathrm{m}$. 


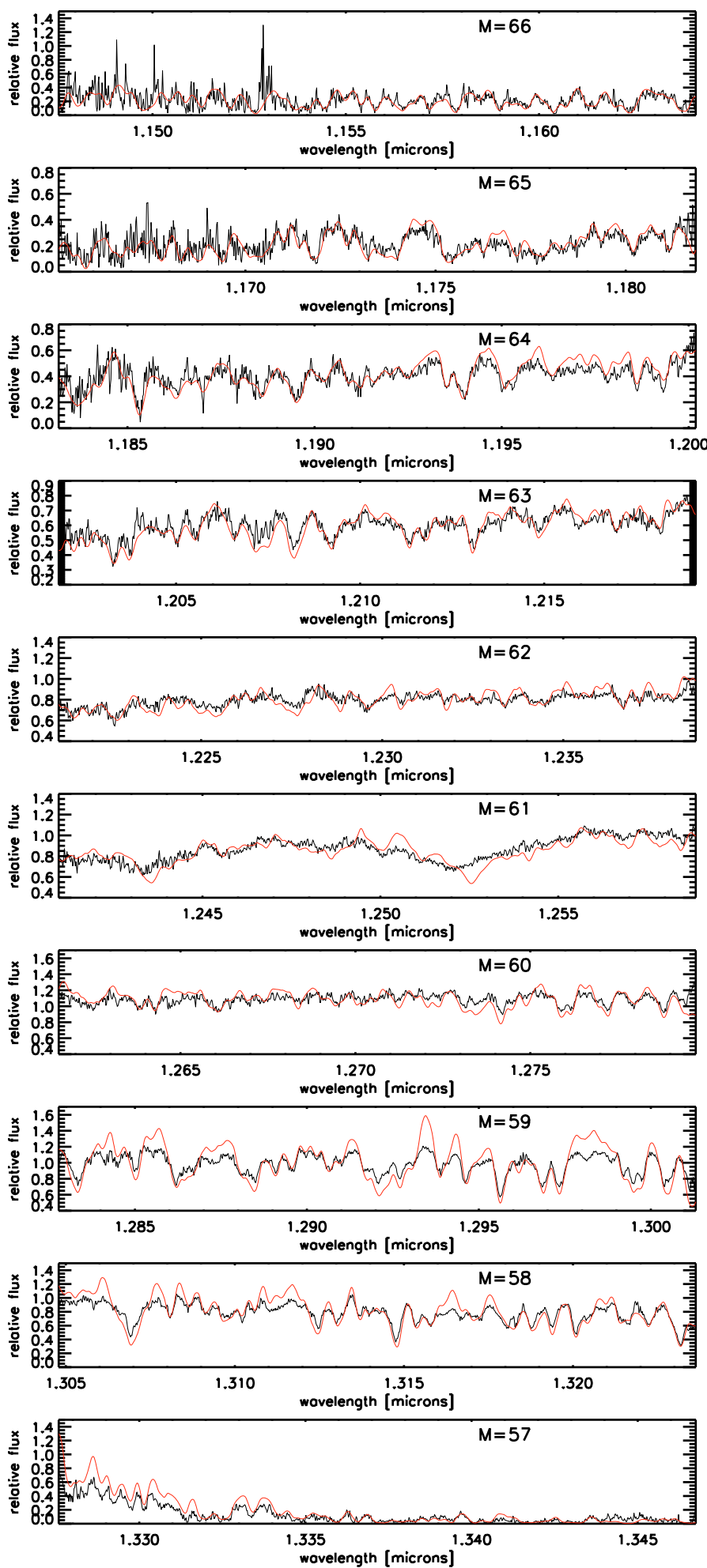

Fig. 8. 2MASS J15031961+2525196. Black and red lines correspond to the observed and modelled spectra, respectively. The model is for an effective temperature $T_{\text {eff }}=1009 \mathrm{~K}$ and $g=10^{4.6} \mathrm{~cm} \mathrm{~s}^{-2}$.

In general, our observed spectra are well reproduced by the best modelled spectra. In a few cases we have found some small differences. This is the case of 2MASS J15031961+2525196 (T5) (see Fig. 8), where the best model is found for $\log g=4.6$ and $T_{\text {eff }}=1009 \mathrm{~K}$. The observed $\mathrm{KI}$ doublet in order 61 is not perfectly reproduced by the model. We also note some differences in orders 59 and 58. Small differences in these

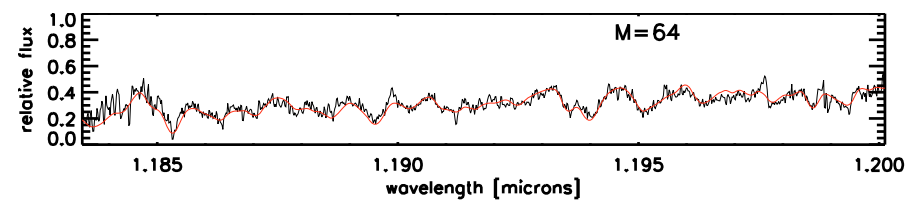

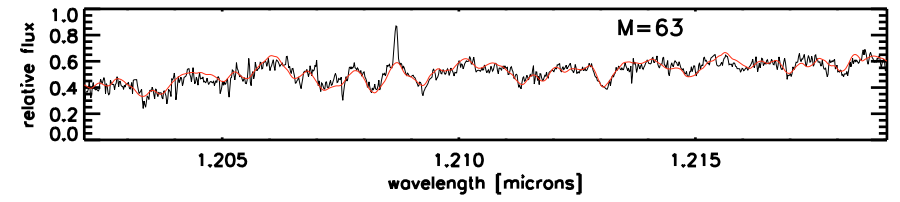
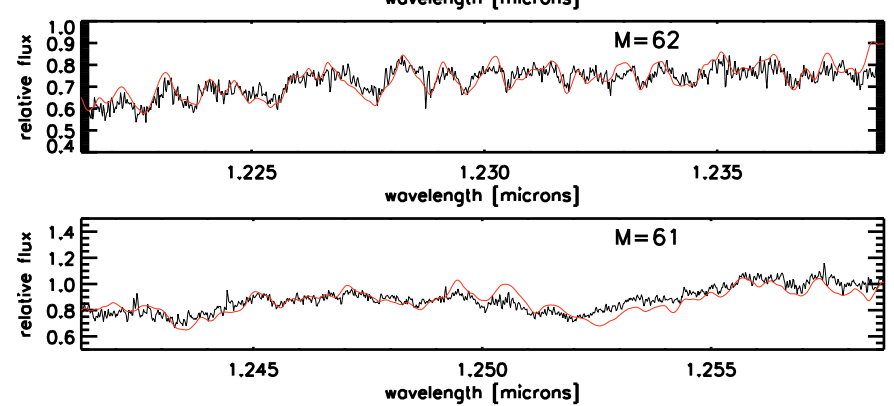

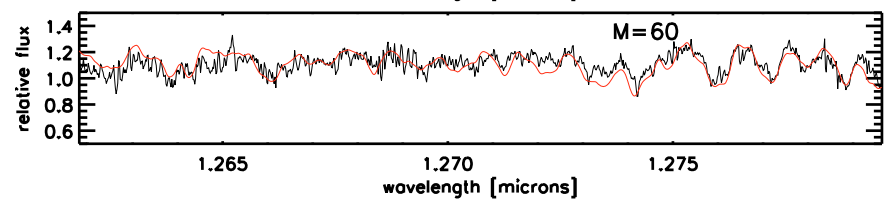
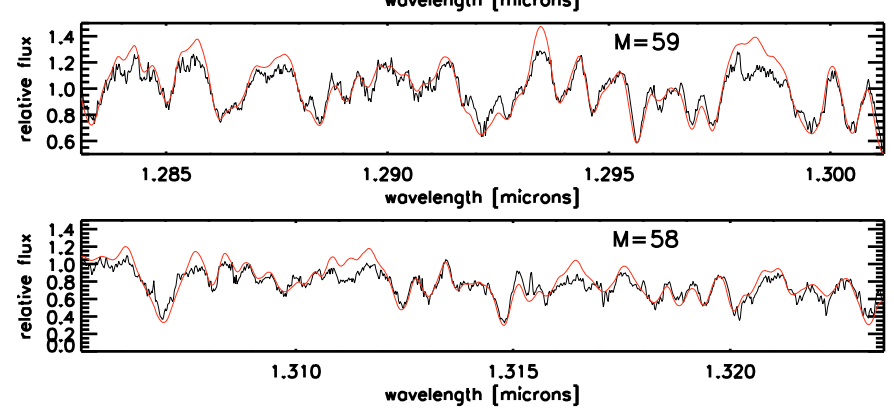

Fig. 9. SDSS J162414.37+002915.6. Black and red lines correspond to the observed and modelled spectra, respectively. The model is for an effective temperature of $980 \mathrm{~K}$ and a surface gravity of $10^{4.8} \mathrm{~cm} \mathrm{~s}^{-2}$.

orders are also present in SDSS J162414.37+002915.6 (T6) (see Fig. 9). These are much smaller in later spectral types (e.g., SDSS J134646.45-003150.4 (T6.5), see Fig. 10). For late $\mathrm{T}$ dwarfs, with faint K I doublets, the models provide an excellent match to the observations for all the orders, with a few exceptions such as the order 61 in 2MASS J12171110-0311131 (see Fig. 12), where the model is flat towards the blue region. We note that the observed spectrum may be affected by errors in the flat-fielding. Note also that there is a faint line of $\mathrm{KI}$ at $1.2525 \mu \mathrm{m}$ in the best modelled spectra for order 61 of GL $570 \mathrm{D}$ that disappears when considering an effective temperature $48 \mathrm{~K}$ lower (see Fig. 13).

The two earlier T dwarfs of our sample, SDSSp J125453.90012247.4 and 2MASS J05591914-1404488, present significant differences between the best modelled and observed spectra. The modelled spectrum corresponding to the average values $\left(T_{\mathrm{eff}}=\right.$ $1002 \mathrm{~K}$ and $\log g=4.9$ ) in 2MASS J05591914-1404488 fails to mimic order 57 with many water vapor lines. The K I doublet at 1.2436 and $1.2525 \mu \mathrm{m}$, very sensitive to temperature, is also difficult to reproduce. Order 57 is clearly better modelled with a higher temperature $T_{\text {eff }}=1700 \mathrm{~K}$. 

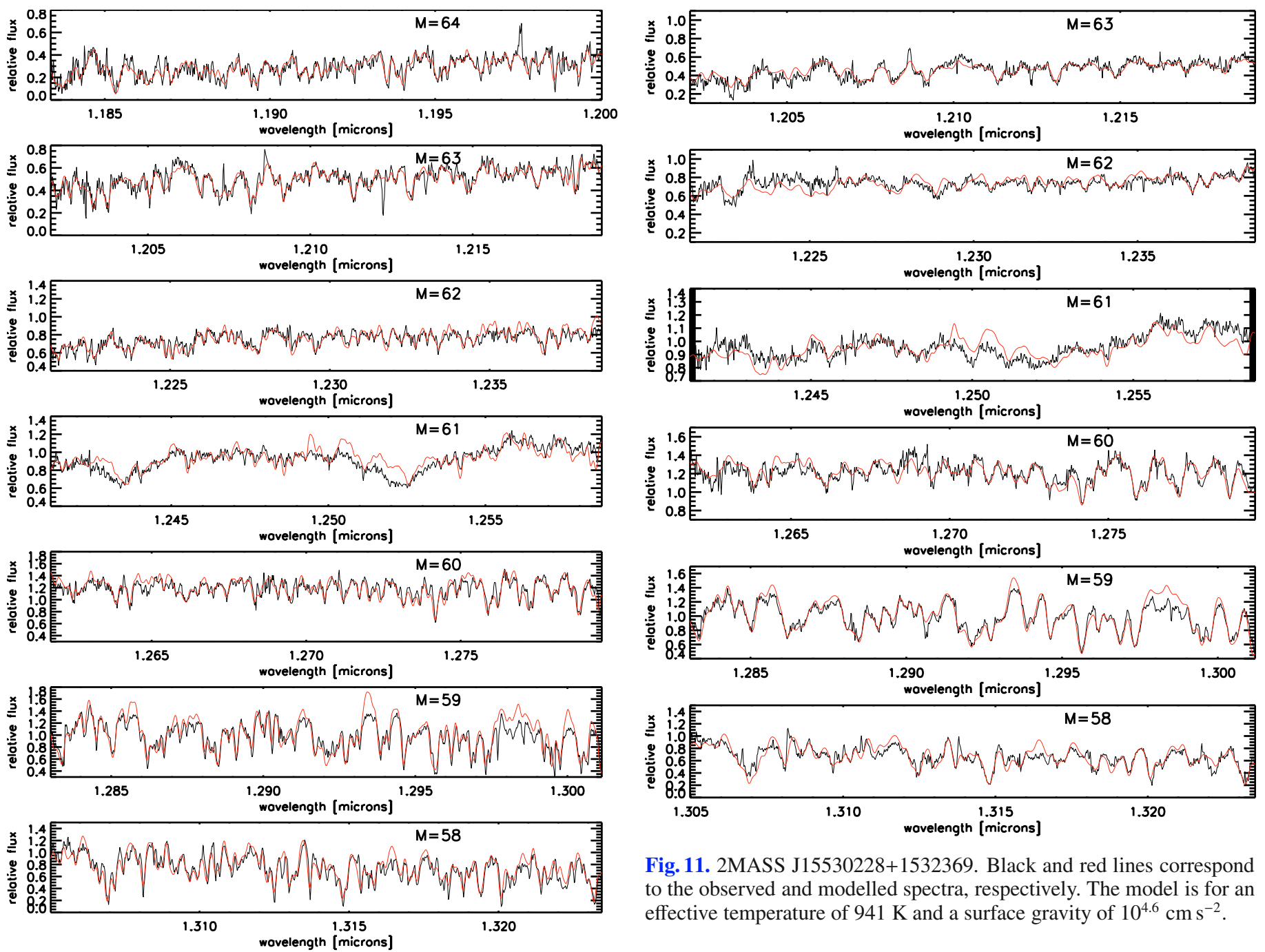

Fig. 11. 2MASS J15530228+1532369. Black and red lines correspond to the observed and modelled spectra, respectively. The model is for an effective temperature of $941 \mathrm{~K}$ and a surface gravity of $10^{4.6} \mathrm{~cm} \mathrm{~s}^{-2}$.

Fig. 10. SDSS J134646.45-003150.4. Black and red lines correspond to the observed and modelled spectra, respectively. The model is for an effective temperature of $990 \mathrm{~K}$ and a surface gravity of $10^{4.1} \mathrm{~cm} \mathrm{~s}^{-2}$.

\subsection{Physical parameters of $T$ dwarfs}

\subsubsection{Rotation, effective temperature and surface gravity}

For our list of nine $\mathrm{T}$ dwarfs, we have determined their projected rotational velocity $\left(v_{\text {rot }} \sin i\right)$, effective temperature and surface gravity from the comparison of the high resolution spectra with the AMES-COND models (see Table 11). The rotational velocities determined here from modelled spectra are in good agreement (i.e., within 1- $\sigma$ uncertainties) with those obtained by ZO06, who used SDSS J134646.45-003150.4 as a non-rotator reference to measure the $v_{\text {rot }} \sin i$ parameter of the sample (i.e., they assumed $v_{\text {rot }} \sin i=0$ for this object). With the exception of 2MASS J12171110-0311131, our values are, indeed, systematically higher by a few $\mathrm{km} \mathrm{s}^{-1}$, which is expected since the template used by ZO06 has a small rotation of $16 \mathrm{~km} \mathrm{~s}^{-1}$ according to our analysis.

The effective temperatures of the $\mathrm{T}$ dwarfs with spectral types later than T5 are between 922 and $1009 \mathrm{~K}$, with errors between $\approx 50$ and $200 \mathrm{~K}$ (see Table 11). The surface gravities of those objects lie between $10^{4.1}$ and $10^{4.9} \mathrm{~cm} \mathrm{~s}^{-2}$, and errors are of $\approx 0.7$ dex. In Sects. 5.2 and 5.3 we discuss our results on $T_{\text {eff }}$ and $\log g$.

\subsubsection{Mass and age}

We have estimated the mass and age of our $\mathrm{T}$ dwarfs using state-of-the-art evolutionary models. Our spectroscopically derived values $\left\langle T_{\text {eff }}\right\rangle$ and $\langle\log g\rangle$ are plotted in the two panels of Fig. 15. The T2V dwarf SDSSp J125453.90-012247.4 is discarded from the figure since the modelled spectra found here do not reproduce the observations satisfactorily. Therefore, only mid- and late-T dwarfs are shown in the $T_{\text {eff }}$ versus $\log g$ diagram, which we remark is independent of the distance to the sources. Figure 15 (top) shows, overplotted on the data, the solar metallicity models by the Lyon group (Baraffe et al. 2003), with isochrones (from $10 \mathrm{Myr}$ to $10 \mathrm{Gyr}$ ) and curves of constant mass $\left(5,10,20,30\right.$, and $\left.50 M_{\mathrm{J}}\right)$. Figure 15 (bottom) shows our data with the solar metallicity models by the Arizona group (Burrows et al. 1997): isochrones (10 Myr, 0.1, 1 and $10 \mathrm{Gyr}$ ) and curves of constant mass $\left(5,10,20,30,50,70\right.$ and $\left.75 M_{\mathrm{J}}\right)$.

Table 11 shows the values of mass and age for our sample of $\mathrm{T}$ dwarfs that are derived from the $T_{\text {eff }}$ versus $\log g$ diagram using the models of the Lyon group. The mass and age uncertainty intervals are inferred from the error bars in the effective temperature and surface gravity. Summarizing, all these objects have a mass in the interval 5-75 $M_{\mathrm{J}}$, thus confirming their very likely substellarity, and likely ages that interestingly seem to be younger than the solar system. We note, however, that considering the large error bars, most of the T dwarfs in our sample may have an age consistent with a few to several Gyr. For GL 570 D and 2MASS J04151954-0935066 we have derived an age upper 
Table 11. Projected rotational velocity, temperature, surface gravity, mass and age of $\mathrm{T}$ dwarfs.

\begin{tabular}{|c|c|c|c|c|c|c|c|c|c|c|}
\hline$\overline{\text { Object }}$ & Spectral type & $\begin{array}{l}v_{\text {rot }} \sin i \\
\mathrm{~km} \mathrm{~s}^{-1} \\
\end{array}$ & $\begin{array}{c}\left\langle T_{\text {eff }}\right\rangle \\
\mathrm{K} \\
\end{array}$ & $\begin{array}{c}\text { Prev. } \\
\mathrm{K} \\
\end{array}$ & Ref. & $\begin{array}{c}\langle\log g\rangle \\
\left(\mathrm{cm} \mathrm{s}^{-2}\right) \\
\end{array}$ & $\begin{array}{c}\text { Prev. } \\
\left(\mathrm{cm} \mathrm{s}^{-2}\right)\end{array}$ & Ref. & $\begin{array}{l}M \\
M_{\mathrm{J}} \\
\end{array}$ & $\begin{array}{l}\text { Age } \\
\text { Gyr }\end{array}$ \\
\hline SDSSp J125453.90-012247.4 & T2 & $34 \pm 7$ & $2007 \pm 662$ & 1200 & 1 & $4.3 \pm 1.0$ & 5.0 & 1 & - & - \\
\hline 2MASS J05591914-1404488 & $\mathrm{T} 4.5$ & $28 \pm 5$ & $1002 \pm 278$ & 1200 & 1 & $4.9 \pm 0.8$ & 5.5 & 1 & $30_{-23}^{+45}$ & $1.0_{-0.9}^{+9}$ \\
\hline 2MASS J15031961+2525196 & T5 & $36 \pm 5$ & $1009 \pm 217$ & 1178 & 2 & $4.6 \pm 0.8$ & $\approx 5.0$ & 5 & $18_{-13}^{+37}$ & $0.4_{-0.3}^{+4.6}$ \\
\hline SDSS J162414.37+002915.6 & T6 & $42 \pm 5$ & $980 \pm 163$ & $1002_{86}^{+98}$ & 3 & $4.8 \pm 0.7$ & $\approx 5.0$ & 5 & $23_{-16}^{+52}$ & $0.6_{-0.5}^{+9.4}$ \\
\hline SDSS J134646.45-003150.4 & T6.5 & $16 \pm 5$ & $990 \pm 107$ & $960-1020$ & 4 & $4.1 \pm 0.4$ & $5.0-5.2$ & 4 & $10_{-5}^{+10}$ & $0.1_{-0.08}^{-0.4}$ \\
\hline 2MASS J15530228+1532369 & $\mathrm{T} 7$ & $30 \pm 7$ & $941 \pm 138$ & 893 & 2 & $4.6 \pm 0.6$ & $\approx 5.0$ & 5 & $18_{-11}^{+30}$ & $0.4_{-0.3}^{+3.68}$ \\
\hline 2MASS J12171110-0311131 & $\mathrm{T} 7.5$ & $29 \pm 7$ & $922 \pm 103$ & $860-880$ & 4 & $4.8 \pm 0.7$ & $4.7-4.9$ & 4 & $23_{-16}^{+52}$ & $0.8_{-07}^{+0.9}$ \\
\hline GL 570D & $\mathrm{T} 7.5$ & $32 \pm 8$ & $948 \pm 53$ & $780-820$ & 4 & $4.5 \pm 0.5$ & 5.1 & 4 & $15_{-9}^{+15}$ & $0.3_{-0.2}^{+1.7}$ \\
\hline 2MASS J04151954-0935066 & $\mathrm{T} 8$ & $36 \pm 7$ & $947 \pm 79$ & $740-760$ & 4 & $4.3 \pm 0.7$ & $4.9-5.0$ & 4 & $10_{-6}^{+20}$ & $0.1_{-0.09}^{+1.9}$ \\
\hline
\end{tabular}

${ }^{1}$ Cushing et al. (2008). ${ }^{2}$ Estimated $T_{\text {eff }}$ derived from the $T_{\text {eff }}$-spectral type relation in Looper et al. (2008). ${ }^{3}$ Vrba et al. (2004). ${ }^{4}$ Burgasser et al. (2006a). ${ }^{5}$ Knapp et al. (2004).
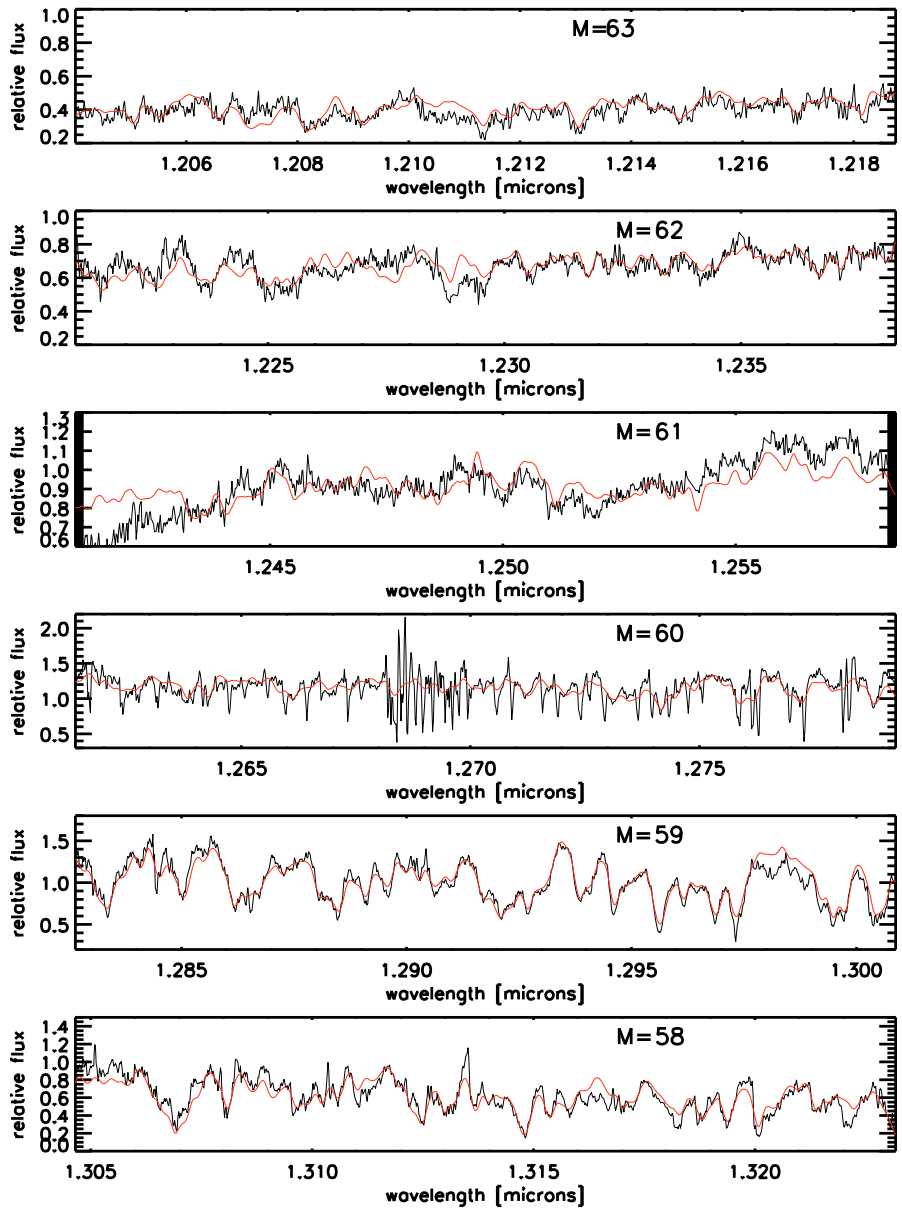

Fig. 12. 2MASS J12171110-0311131. Black and red lines correspond to the observed and modelled spectra, respectively. The model is for an effective temperature of $922 \mathrm{~K}$ and a surface gravity of $10^{4.8} \mathrm{~cm} \mathrm{~s}^{-2}$.

limit of 2 Gyr, and only for SDSS J134646.45-003150.4 the upper limit is below $1 \mathrm{Gyr}$.

\section{Discussion}

\subsection{Modelled and observed spectra}

Most of the lines in a $\mathrm{T}$ dwarf are due to water vapor. A few atomic lines can be identified, but some of them, e.g. the $\mathrm{Rb} \mathrm{I}$ line, are very weak compared to the haze of molecular lines.
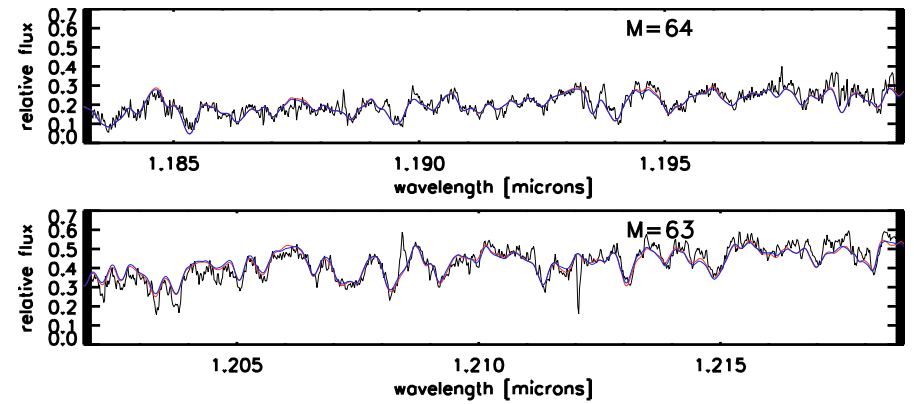

1.225
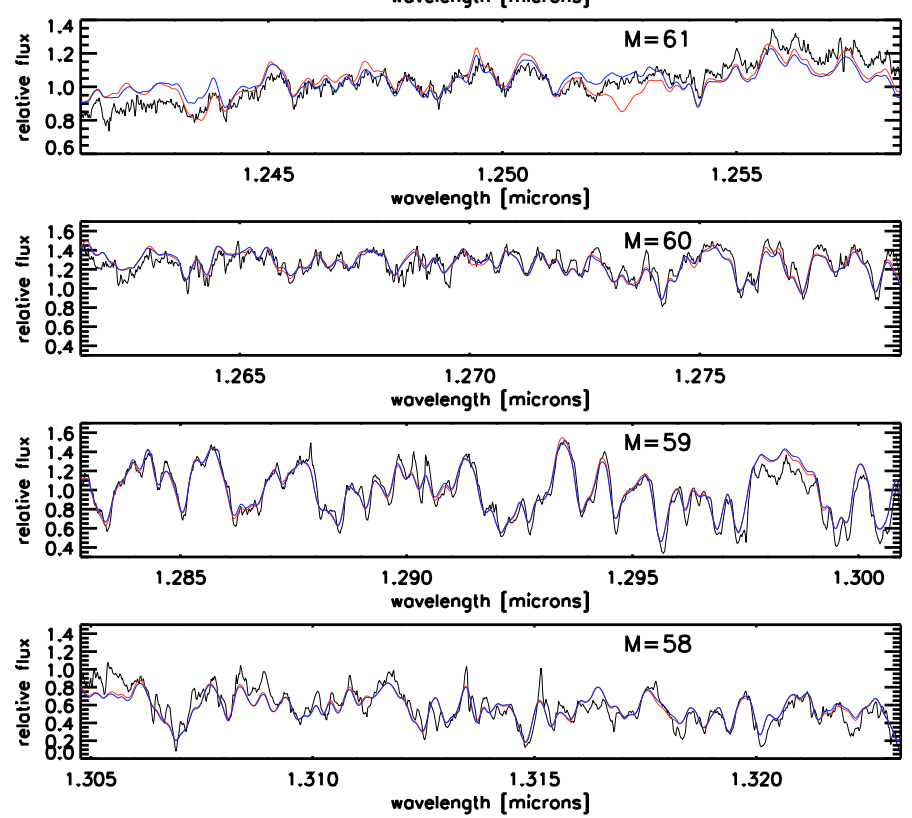

Fig. 13. GL 570 D. Black line corresponds to the observed spectrum, and red and blue lines correspond to modelled spectra with the same $g=10^{4.5} \mathrm{~cm} \mathrm{~s}^{-2}$ and $T_{\text {eff }}=948 \mathrm{~K}$ and $900 \mathrm{~K}$.

In general, the modelled spectra match remarkably well the high-resolution observed spectra. We note only that the profiles of the strong KI lines are not so well matched by the models. This is likely due to the approximations used for the intrinsic line profiles in the models, most importantly estimates of the 

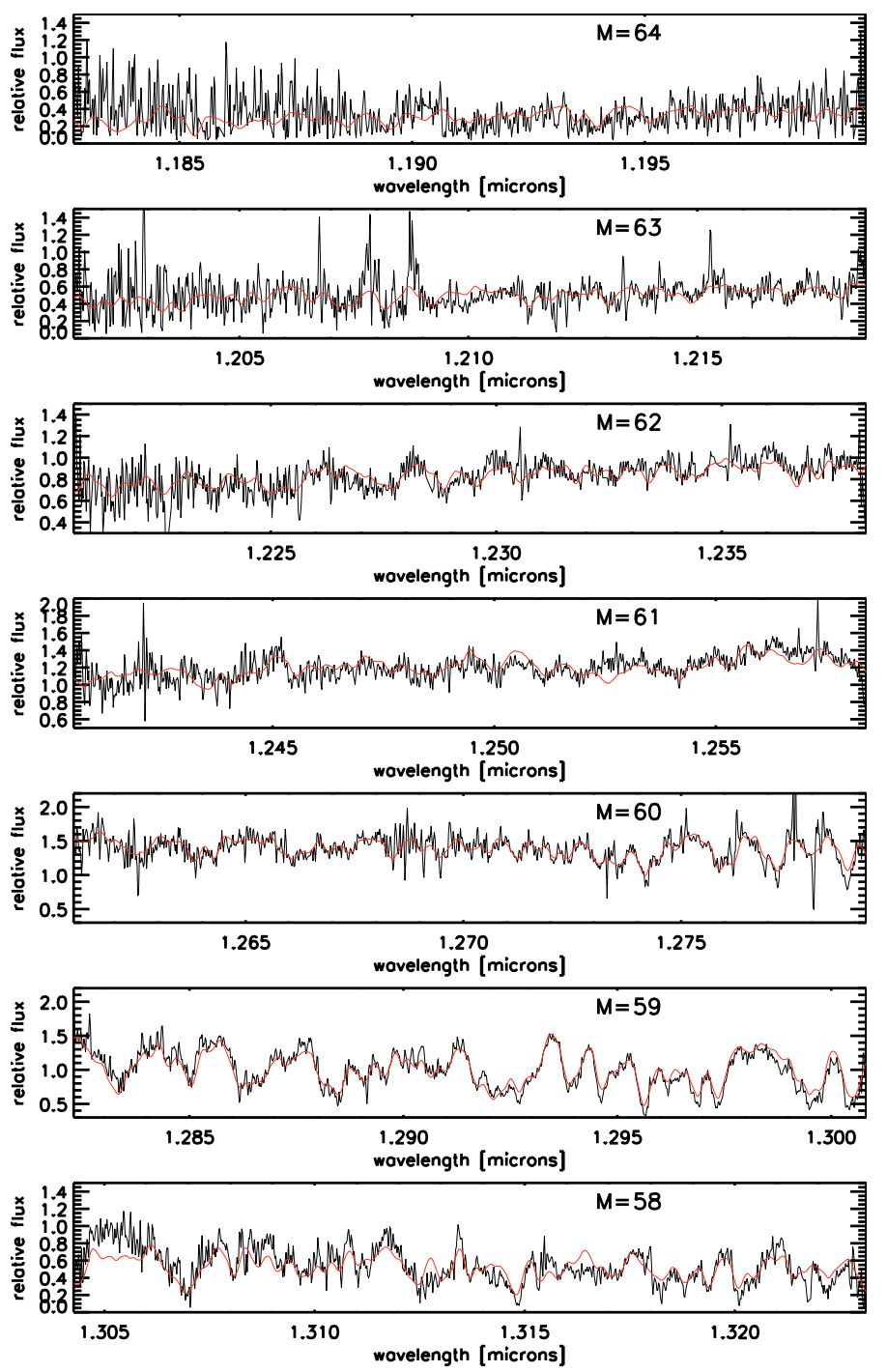

Fig. 14. 2MASS J04151954-0935066. Black and red lines correspond to the observed and modelled spectra, respectively. The model is for an effective temperature of $947 \mathrm{~K}$ and a surface gravity of $10^{4.3} \mathrm{~cm} \mathrm{~s}^{-2}$.

damping constants. For example, Allard et al. (2003) and Johnas et al. (2007) have demonstrated the importance of detailed line profiles (not just damping constants) for optical alkali resonance lines in cool sub-stellar objects, however, such profiles are not yet available for the IR alkali lines. Therefore, the profiles of these atomic lines cannot be modelled accurately, and so cannot be used to obtain reliable parameter estimates. From qualitative considerations, however, the models predict that for surface temperatures of 900-1000 K, the atomic lines of K I strengthen with decreasing gravity, as was pointed out by Knapp et al. (2004). SDSS J134646.45-003150.4 shows the K I doublet at $1.25 \mu \mathrm{m}$, in contrast to SDSS J162414.37+002915.6 and 2MASS J15530228+1532369, which have similar spectral types. From our analysis, we have found the smallest $\log g$ for SDSS J134646.45-003150.4 in our sample. This is also the object with the lowest estimated age.

The FeH lines are difficult to model, in part due to their relatively poorly known oscillator strength and possible errors in the chemistry. The fact that they become weaker with lower effective temperatures (like those of our sample) is due to the condensation of iron, which reduces the number density of $\mathrm{FeH}$, resulting
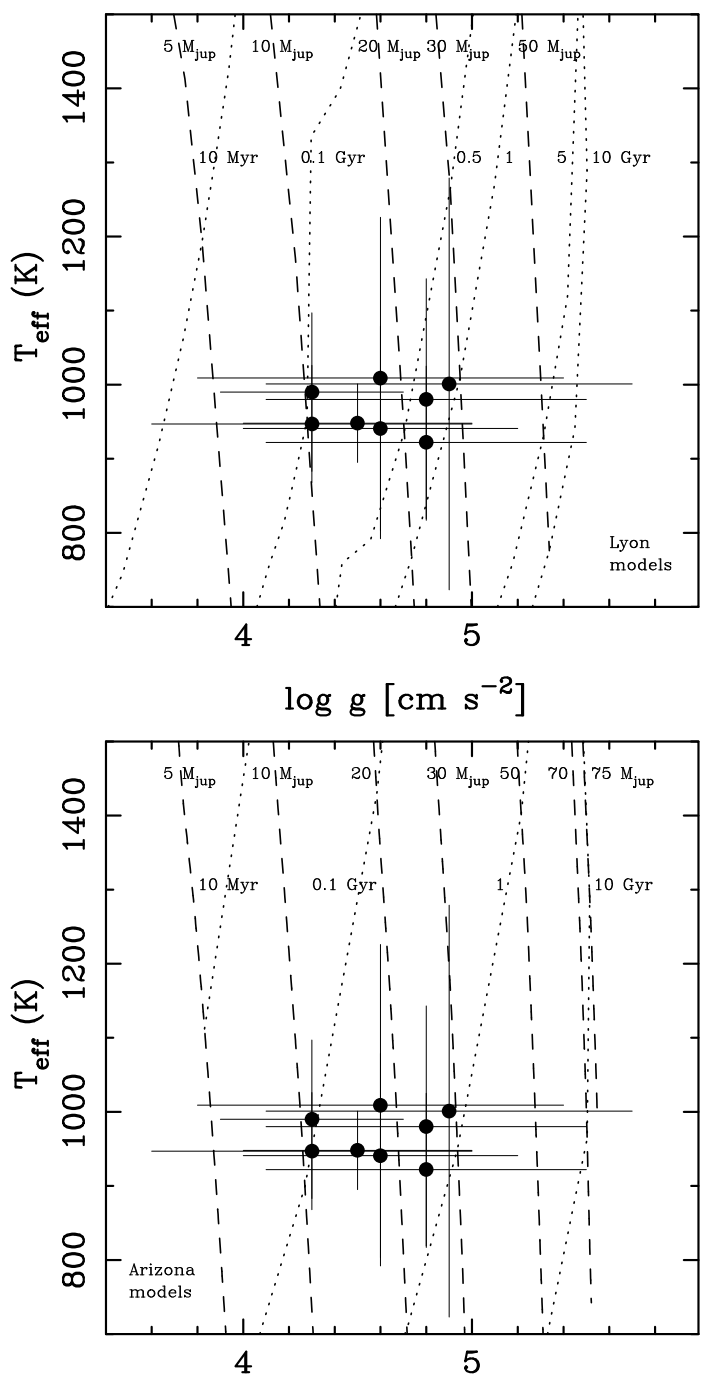

$\log \mathrm{g}\left[\mathrm{cm} \mathrm{s}{ }^{-2}\right]$

Fig. 15. Diagram $T_{\text {eff }}$ versus $\log g$ with the values we have obtained for our sample of T dwarfs (SDSSp J125453.90-012247.4 is not included) and the solar metallicity models by Lyon (top) and Arizona (bottom). The models provide isochrones from $10 \mathrm{Myr}$ to $10 \mathrm{Gyr}$ (dashed lines) and curves of constant mass (dotted lines).

in an important test of the treatment of condensation in the equation of state.

$\mathrm{CH}_{4}$ bands are expected and have been observed in $\mathrm{T}$ dwarfs (Burgasser et al. 2002a) but, in the spectral range considered here, are much weaker than the water vapor lines and so cannot be identified with confidence.

At higher effective temperatures, as for SDSSp J125453.90012247.4 (T2) and 2MASS J05591914-1404488 (T4.5), the models appear to be generally less consistent with the observations. This is likely due to the presence of remnants of dust clouds, floating around in upper layers, that are not included in the modelled spectra (see Ruiz et al. 1997; Ackerman \& Marley 2001; Burgasser et al. 2002b; Burrows et al. 2006; Cooper et al. 2003; Helling et al. 2008). Cushing et al. (2008) have computed the properties of SDSSp J125453.90-012247.4 and 2MASS J05591914-1404488 from the comparison of low and intermediate resolution spectra in the $0.95-14.5 \mu \mathrm{m}$ wavelength range and synthetic spectra. They found that SDSSp J125453.90-012247.4 has condensate clouds that are thicker than those in 2MASS 


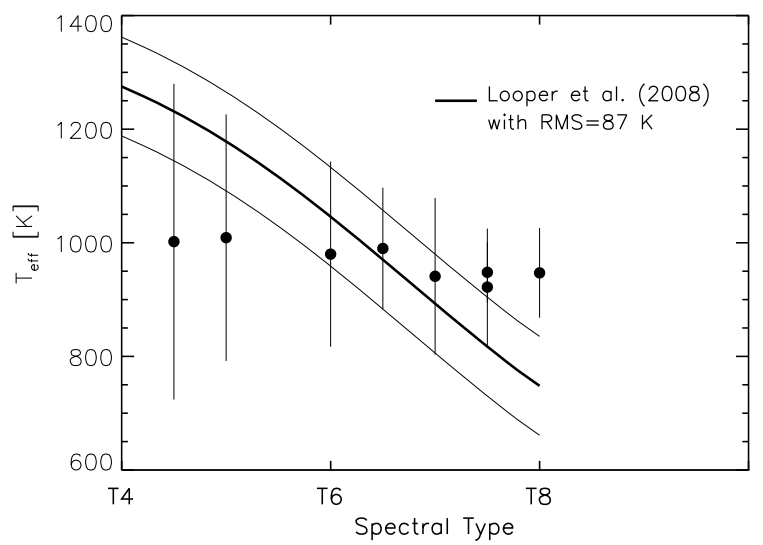

Fig. 16. $T_{\text {eff }}$ versus spectral type for our $\mathrm{T}$ dwarfs (only SDSSp J125453.90-012247.4 is not included) and the $T_{\text {eff }}$-spectral type relation of Looper et al. (2008).

J05591914-1404488, which may explain the difference in the spectra of these overluminous $\mathrm{T}$ dwarfs. Cushing et al. also discussed the possible unresolved binarity in both objects. They determined $T_{\text {eff }} \sim 1200 \mathrm{~K}(<1150 \mathrm{~K}), \log g \sim 4.8(<5.38)$ and an age of $\sim 0.4(<10)$ Gyr for 2MASS J05591914-1404488 assuming it is a single object (equal mass binary). The same temperature of $\sim 1200 \mathrm{~K}$ is found for SDSSp J125453.90-012247.4. Our values of $T_{\text {eff }}, \log g$ and age of 2MASS J05591914-1404488 (see Table 11) are in agreement with those of Cushing et al.

We will present a further study of the effects of the metallicity on 2MASS J05591914-1404488 using a new set of synthetic models (del Burgo et al., in preparation). The new models will include a completely new equation of state and (where possible and available) improved line data. Preliminary tests with the new equation of state show great improvements in the $M$ dwarf regime. In addition, a physical model for the dust cloud formation coupled to the structure of the atmosphere is being developed; this is required at least for the transition $\mathrm{T} \rightarrow \mathrm{L} \rightarrow \mathrm{M}$.

\subsection{Effective temperature}

For the two earliest T dwarfs, 2MASS J05591914-1404488 and especially SDSSp J125453.90-012247.4, we found that the models used here cannot reproduce the observed spectra so well. The T4.5 dwarf 2MASS J05591914-1404488 has historically been considered enigmatic (see Vrba et al. 2004). Our best modelled spectra with $\left\langle T_{\text {eff }}\right\rangle$ of $1002 \pm 278 \mathrm{~K}$ is, taking into account the errors, consistent with the estimate of $1231 \mathrm{~K}$ from the relation of Looper et al. and the value of $1200 \mathrm{~K}$ found by Cushing et al. (2008). Our best modelled spectrum of SDSSp J125453.90-012247.4 indicates an effective temperature above $2000 \mathrm{~K}$, which is much higher than that found by Vrba et al. (2004) and Cushing et al. (2008), but consistent (i.e. within the errors) with a temperature around $1500 \mathrm{~K}$. This value is similar to the estimate of $1370 \mathrm{~K}$ in the relation of Looper et al.

We also find some differences for the latest $\mathrm{T}$ dwarfs. Our values of $\left\langle T_{\text {eff }}\right\rangle$ for GL 570 D and 2MASS J04151954-0935066 are about $150 \mathrm{~K}$ higher than those from the literature (see Table 11). Geballe et al. (2001) use $R=400$ spectra and accurate photometry of GL 570D to determine that $T_{\text {eff }}$ of 784-824 K and $\log g=5.00-5.27\left(\mathrm{~cm} \mathrm{~s}^{-2}\right)$, assuming an age of 2-5 Gyr.

Figure 16 shows $T_{\text {eff }}$ versus spectral type for the eight latest $\mathrm{T}$ dwarfs of our sample, and the $T_{\text {eff }}$-spectral type relation found in Looper et al. (2008). Our values appear to be rather flat from T4.5 down to T8, which clearly contrasts with the trend
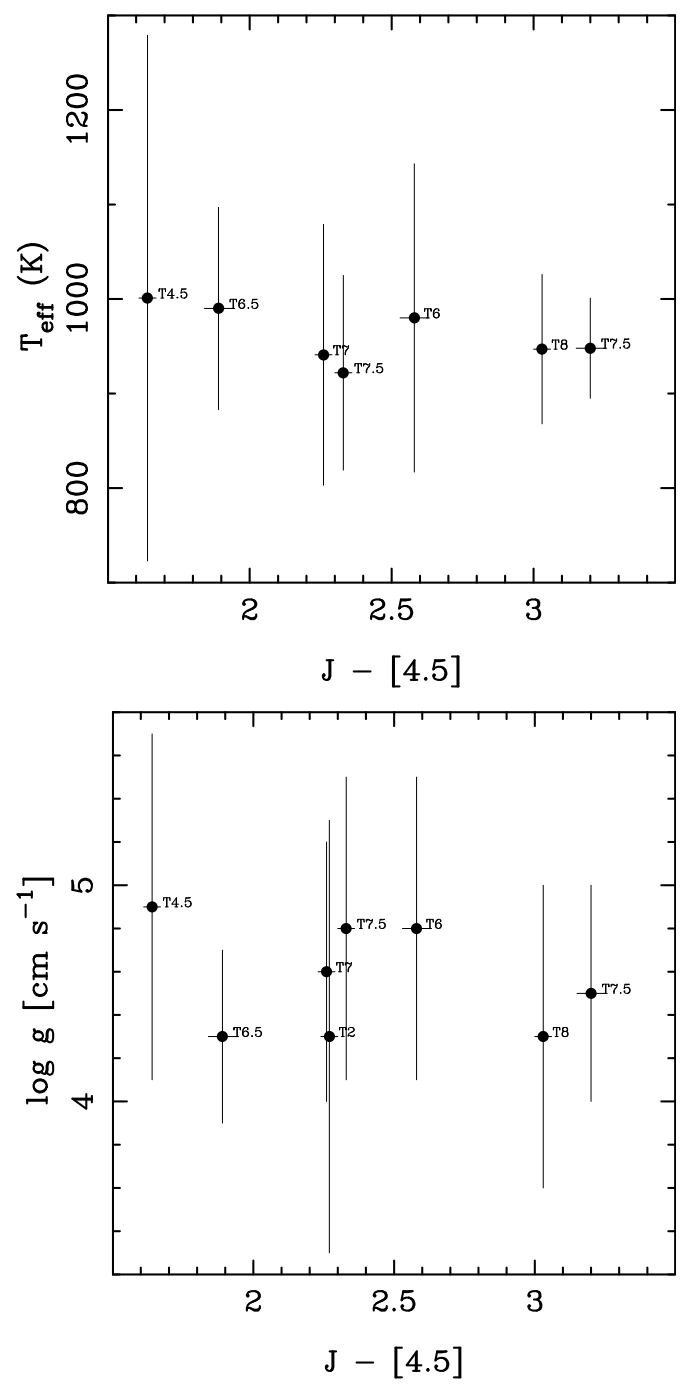

Fig. 17. $T_{\text {eff }}$ versus colour $J-[4.5]$ (top) and $\log g$ versus $J-[4.5]$ (bottom).

delineated by Looper et al. (2008). We note, however, that such a trend is still compatible with our measurements if error bars are taken into account. The apparent constant temperature derived in our work may be due to a degeneration in the method (synthetic and observed dataset) that, despite the high spectral resolution of the observations, is not accurately sensitive to $T_{\text {eff }}$ and $\log g$, partly due to the small wavelength range coverage of the data. Cushing et al. (2008) found that the values of $T_{\text {eff }}$ obtained from $J$-band low-resolution spectra are consistent with those derived from fitting the full SED, using a model grid with temperature steps of $100 \mathrm{~K}$.

Figure 17 (top) shows $\left\langle T_{\text {eff }}\right\rangle$ as a function of the colour $J-$ [4.5] for the T dwarfs later than T4.5 (photometric data are compiled from Patten et al. 2006). These objects display a wide range of colours ( $\sim 2 \mathrm{mag}$ ), however, our derived temperatures differ by less than $100 \mathrm{~K}$. It seems difficult to reconcile such a large colour range with just one value of temperature, unless other atmospheric parameters (like metallicity, cloud coverage, and others) are taken into account.

\subsection{Surface gravity}

Our values of $\log g$ are in good agreement with those of Knapp et al. (2004, see Table 11), whose $\log g$ values were derived from 
the comparison of observed $H-K$ colors to model-predicted $H-K$ colors. The largest difference is for the T8 dwarf $2 \mathrm{MASS}$ J04151954-0935066, where the estimate of Knapp et al. (2004) is 0.7 dex higher than our value. Figure 17 (bottom) shows $\log g$ versus infrared colour $J-[4.5]$, where no obvious trend is apparent.

\subsection{Mass and age}

The estimated masses in the T dwarfs of our sample are in good agreement with the model fit values of Burgasser et al. (2004) for three of the common objects (SDSS J162414.37+002915.6, 2MASS J15530228+1532369, 2MASS J04151954-0935066). The only exception is GL570 D. Burgasser et al. (2004) find a mass of $<0.001 M_{\odot}$, i.e., $<1 M_{\mathrm{J}}$, and also provide a expected value of 30-50 $M_{\mathrm{J}}$. We find a value of $15 M_{\mathrm{J}}$, with an upper limit of $30 M_{\mathrm{J}}$ that is the same as the lower expected value given by Burgasser et al. Our lower limit $\left(6 M_{\mathrm{J}}\right)$ is several times higher than the fitted value of Burgasser et al. (2004).

Our result on the apparent young age of the field T dwarfs (see Table 11) is in agreement with recent kinematical studies based on proper motions and space velocities by Bannister \& Jameson (2007) and Zapatero Osorio (2007). The latter authors found that about $40 \%$ of the L and T-type population of the solar neighborhood may have an age close to that of the Hyades cluster (around $600 \mathrm{Myr}$ ), and that the brown dwarf population is kinematically younger than solar-type to early-M stars with likely ages in the interval $0.5-4$ Gyr. We also note that our upper limit of 2 Gyr for the widely studied object GL 570 D agrees with the lower limit assumed by Geballe et al. (2001).

\section{Conclusions}

We conclude that the high resolution spectra corresponding to $\mathrm{T}$ dwarfs with spectral types later than $\mathrm{T} 5$ are well reproduced by the AMES-COND solar metallicity models provided by the PHOENIX code. The models reproduce in detail many faint absorption features in the high resolution $J$-band spectra, which are mainly due to water vapor. There are also strong K I lines, which turn out to be more difficult to model due to uncertain damping constants. The temperature and surface gravity determined from the comparison of the modelled and observed spectra are consistent with those found in the literature. We find a marked flat behaviour of $T_{\text {eff }}$ with spectral type, although a possible gradient is compatible with the errors. High resolution spectroscopy $(R \sim 20000)$ in the $J$-band and AMES-COND models seems to be insufficient to show the existence of a possible gradient in effective temperature from early to late $\mathrm{T}$ dwarfs. The comparison between the spectroscopically derived $T_{\text {eff }}$ and $\log g$ of our targets and the evolutionary models by the Lyon and Arizona groups yields ages in the range 0.1-5 Gyr and masses between $\sim 5$ and $75 M_{\mathrm{J}}$ for the target sample with spectral types $\geq T 5$. For the earlier type dwarfs the spectral models do not provide suitable fits, which is likely due to the presence of condensate clouds that are not incorporated in the models.

Acknowledgements. The authors would like to thank the referee for useful comments and Carlos Allende for lively discussions. The data used here were obtained at the W. M. Keck Observatory, which is operated as a scientific partnership between the California Institute of Technology, the University of California, and the National Aeronautics Space Administration. Support for this project has been provided by the Spanish Ministry of Science via project AYA2007-67458 and by a NASA-Keck analysis grant provided by the Jet Propulsion Laboratory. This work was also supported by the DFG via Graduiertenkolleg 1351. Some of the calculations presented here were performed at the Höchstleistungs
Rechenzentrum Nord (HLRN); at the NASA's Advanced Supercomputing Division's Project Columbia, at the Hamburger Sternwarte Apple G5 and Delta Opteron clusters financially supported by the DFG and the State of Hamburg; and at the National Energy Research Supercomputer Center (NERSC), which is supported by the Office of Science of the US Department of Energy under Contract No. DE-AC03-76SF00098. We thank all these institutions for the generous allocation of computer time.

\section{References}

Ackerman, A. S., \& Marley M. S. 2001, ApJ, 556, 872

Allard, F., Hauschildt, P. H., Baraffe, I., \& Chabrier, G. 1996, ApJ, 465, L123

Allard, F., Hauschildt, P. H., Alexander, D. R., \& Starrfield, S. 1997, ARA\&A, 35,137

Allard, F., Hauschildt, P., \& Schwenke, D. 2000, ApJ, 540, 1005

Allard, F., Hauschildt, P. H., Alexander, D. R., Tamanai, A., \& Schweitzer, A. 2001, ApJ, 556, 357

Allard, N. F., Allard, F., Hauschildt, P. H., Kielkopf, J. F., \& Machin, L. 2003, A\&A, 411, L473

Bannister, N. P., \& Jameson, R. F. 2007, MNRAS, 378, L24

Baraffe, I., Chabrier, G., Barman, T. S., Allard, F., \& Hauschildt, P. H. 2003, A\&A, 402, 701

Barber, R. J., Tennyson, J., Harris, G. J., \& Tolchenov, R. N. 2006, MNRAS, 368,1087

Burgasser, A. J., Kirkpatrick, J. D., Brown, M. E., et al. 1999, ApJ, 522, L65 Burgasser, A. J., Kirkpatrick, J. D., Brown, M. E., et al. 2002a, ApJ, 564, 421 Burgasser, A. J., Marley, M. S., Ackerman, A. S., et al. 2002b, ApJ, 571, 151 Burgasser, A. J., Kirkpatrick, J. D., McGovern, M. R., et al. 2004, ApJ, 604, 827 Burgasser, A. J., Geballe, T. R., Leggett, S. K., Kirkpatrick, J. D., \& Golimowski, D. A. 2006a, ApJ, 637, 1067

Burgasser, A. J., Burrows, A., \& Kirkpatrick, J. D. 2006b, ApJ, 639, 1095 Burrows, A., Marley, M. S., Hubbard, W. B., et al. 1997, ApJ, 491, 856 Burrows, A., Marley, M. S., \& Sharp, C. M. 2000, ApJ, 531, 438

Burrows, A., Sudarsky, D., \& Hubeny, I. 2006, ApJ, 640, 1063

Cooper, C. S., Sudarsky, D., Milsom, J. A., Lunine, J. I., \& Burrows, A. 2003, 586,1320

Cuby, J. G., Saracco, P., Moorwood, A. F. M., et al. 1999, A\&A, 349, L41 Cushing, M. C., Marley, M. S., Saumon, D., et al. 2008, ApJ, 678, 1372 Delorme, P., Delfosse, X., Albert, L., et al. 2008, A\&A, 482, 961

Dulick, M., Bauschlicher, C. W. Jr., Burrows, A., et al. 2003, ApJ, 594, 651 Fegley, B. Jr., \& Lodders, K. 1996, ApJ, 472, L37

Geballe, T. R., Saumon, D., Legget, S. K., et al. 2001, ApJ, 556, 373

Gray, D. F. 1992, The Observations and Analysis of Stellar Photospheres (Cambridge University Press), 2nd. edn.

Griffith, C. A., Yelle, R. V., \& Marley, M. S. 1998, Science, 282, 5396

Hauschildt, P. H. 1992, JQSRT, 47, 433

Hauschildt, P. H. 1993, JQSRT, 50, 301

Hauschildt, P. H., \& Baron, E. 1999, JCoAM, 109, 41

Helling, Ch., Ackerman, A., Allard, F., et al. 2008, MNRAS, 391, 1854

Johnas, C. M. S., Hauschildt, P. H., Schweitzer, A., et al. 2007, A\&A, 475, 1039

Jones, H. R. A., Pavlenko, Y., Viti, S., et al. 2005, MNRAS, 358, 105

Knapp, G. R., Leggett, S. K., Fan, X., et al. 2004, ApJ, 127, 3553

Leggett, S. K., Marley, M. S., Freedman, R., et al. 2007, ApJ, 667, 537

Looper, D. L., Gelino, C. R., Burgasser, A. J., \& Kirkpatrick, J. D. 2008, ApJ, 685,1183

Oppenheimer, B. R., Kulkarni, S. R., Matthews, K., \& Nakajima, T. 1995, Science, 270, 1478

Marley, M. S., Saumon, D., Guillot, T., et al. 1996, Science, 272, 1919

Martín, E. L., \& Zapatero Osorio, M. R. 2003, ApJ, 593, L113

McLean, I. S., Prato, S., McGovern, M. R., et al. 2007, ApJ, 658, 1217

Patten, B. M., Stauffer, J. R., Burrows, A., et al. 2006, ApJ, 651, 502

Reid, I. N. 1999, MNRAS, 302, L21

Ruiz, M. T., Leggett, S. K., \& Allard, F. 1997, ApJL, 491, 107

Saumon, D., Marley, M. S., Cushing, M. C., et al. 2006, ApJ, 647, 552

Saumon, D., Marley, M. S., Leggett, S. K., et al. 2007, ApJ, 656, 1136

Strauss, M. A., Fan, X., Gunn, J. E., et al. 1999, ApJ, 522, L61

Tsuji, T. 2005, ApJ, 621, 1033

Tsuji, T., Ohnaka, K., Aoki, W., \& Nakajima, T. 1996, A\&A, 308, L29

Tsuji, T., Ohnaka, K., \& Aoki, W. 1999, ApJ, 520, L119

Tsuji, T., Nakajima, T., \& Yanagisawa, K. 2004, ApJ, 607, 511

Vrba, F. J., Henden, A. A., Luginbuhl, C. B., et al. 2004, AJ, 127, 2948

Warren, S. J., Henden, A. A., Luginbuhl, C. B., et al. 2007, MNRAS, 381, 1400

Zapatero Osorio, M. R., Martín, E. L., Bouy, H., et al. 2006, ApJ, 647, 1405 (ZO06)

Zapatero Osorio, M. R., Martín, E. L., Béjar, V. J. S., et al. 2007, ApJ, 666, 1205, (ZO07) 